Louisiana State University

LSU Digital Commons

$4-1-2010$

\title{
Strict lyapunov function constructions under lasalle conditions with an application to lotka-volterra systems
}

Frédéric Mazenc

INRAE's Occitanie-Montpellier Centre

Michael Malisoff

Louisiana State University

Follow this and additional works at: https://digitalcommons.Isu.edu/mathematics_pubs

\section{Recommended Citation}

Mazenc, F., \& Malisoff, M. (2010). Strict lyapunov function constructions under lasalle conditions with an application to lotka-volterra systems. IEEE Transactions on Automatic Control, 55 (4), 841-854. https://doi.org/10.1109/TAC.2010.2041995

This Article is brought to you for free and open access by the Department of Mathematics at LSU Digital Commons. It has been accepted for inclusion in Faculty Publications by an authorized administrator of LSU Digital Commons. For more information, please contact ir@lsu.edu. 


\title{
Strict Lyapunov Function Constructions Under LaSalle Conditions with an Application to Lotka-Volterra Systems
}

\author{
Frédéric Mazenc Michael Malisoff
}

\begin{abstract}
We provide new techniques for building explicit global strict Lyapunov functions for broad classes of periodic time varying nonlinear systems satisfying LaSalle conditions. Our new constructions are simpler than the designs available in the literature. We illustrate our work using the Lotka-Volterra model, which plays a fundamental role in bioengineering. We use our strict Lyapunov constructions to prove robustness of the Lotka-Volterra tracking dynamics under uncertainty in the death rates.
\end{abstract}

\section{INTRODUCTION}

Lyapunov functions provide vital tools for the analysis of, and controller design for, nonlinear systems [8], [9], [17]. The two main types of Lyapunov functions are strict Lyapunov functions (also called strong Lyapunov functions, having negative definite time derivatives along trajectories) and nonstrict Lyapunov functions (also called weak Lyapunov functions, whose time derivatives along the trajectories are negative semidefinite); see Section II below for precise definitions.

Strict Lyapunov functions are typically far more useful than nonstrict ones. In general, nonstrict Lyapunov functions can only be used to prove asymptotic stability, using, e.g., the LaSalle invariance

Mazenc is with EPI MERE INRIA-INRA, UMR Analyse des Systèmes et Biométrie, INRA, 2, pl. Viala, 34060 Montpellier, France, mazenc@supagro.inra.fr, Tel: 33 (0) 4996124 98, Fax: 33 (0) 4675214 27. Malisoff is with the Department of Mathematics, Louisiana State University, Baton Rouge, LA 70803-4918 USA, malisoff@1su.edu, Tel: (225) 578-6714, Fax: (225) 578-4276. Malisoff was supported by NSF/DMS Grants 0424011 and 0708084. 
principle, while strict Lyapunov functions can often be used to show robustness properties, such as input-to-state stability (ISS). Robustness is essential in engineering, largely due to uncertainty in dynamical models and noise entering into controllers. For this reason, it is important to construct strict Lyapunov functions, even for systems that are already known to be UGAS.

Moreover, many controller methods (e.g., backstepping [9], forwarding [15], [17] and universal stabilizing controllers [18]) use strict Lyapunov functions. For example, if $V$ is a strict Lyapunov function for a system $\dot{x}=f(t, x)$ for which $\alpha(x):=\inf _{t}\left\{-\left[V_{t}(t, x)+V_{x}(t, x) f(t, x)\right]\right\}$ is radially unbounded, with $f$ and $g$ locally Lipschitz, and with $V, f$, and $g$ all periodic in $t$ with the same period $T$, then $\dot{x}=f(t, x)+g(t, x)[K(t, x)+d]$ is input-to-state stable if $K(t, x)=$ $-\left[V_{x}(t, x) g(t, x)\right]^{\top}$. Consequently, when a global strict Lyapunov function is known, many important stabilization problems can be solved almost immediately.

In general, it is much easier to construct nonstrict Lyapunov functions, owing to the more restrictive decay condition for strict Lyapunov functions. For instance, when a passive nonlinear system is stabilized by linear output feedback, the energy (i.e., storage) function can typically be used as the weak Lyapunov function. This fact is useful for electro-mechanical systems. When a system is stabilized via the Jurdjevic-Quinn theorem, nonstrict Lyapunov functions are typically available, e.g., using the Hamiltonian for Euler Lagrange systems [5], [7], [12], [16]. If a system is known to be UGAS, then converse Lyapunov function theory typically guarantees the existence of a strict Lyapunov function. However, the Lyapunov functions provided by converse theory are often abstract and nonexplicit, and therefore may not always lend themselves to applications. This has motivated a significant literature on constructing strict Lyapunov functions, e.g., [1], [5].

In this work, we present two new strict Lyapunov function constructions, based on transforming nonstrict Lyapunov functions into strict ones, under Lie derivative conditions. The assumptions for our first construction are more general than those of [14] and different from those of [11, Corollary 2]. This is because we allow periodic time varying systems, including cases where all of the higher 
order Lie derivatives are allowed to vanish at some points outside the equilibrium, on some time intervals. Our construction is simpler than the one in [14], even in the special case of time invariant systems.

Our second result uses the Matrosov approach. In general, Matrosov's method can be difficult to apply, because one needs to find the necessary auxiliary functions. Here we give simple sufficient conditions leading to a systematic design of auxiliary functions. The auxiliary functions we construct differ from the ones that are implicitly given in [11], and they lead to strict Lyapunov functions using the Matrosov construction from [13]. In fact, our strict Lyapunov functions have the property that their Lie derivatives are frequently bounded above by negative definite quadratic functions. Another important feature of our work is that it applies to cases where the state space of the system is a general subset of Euclidean space, rather than the whole Euclidean space. This is desirable for biological systems, whose state spaces are often restricted by the requirement that physical quantities need to be nonnegative. We illustrate our approach using an error dynamics associated with the Lotka-Volterra system, which plays a fundamental role in bioengineering.

\section{Definitions And Assumptions}

Throughout this work, $\mathcal{X}$ is any open subset of $\mathbb{R}^{n}$ containing the origin. Consider a nonlinear time varying dynamics

$$
\dot{x}=f(t, x), \quad x \in \mathcal{X}
$$

where $f:[0, \infty) \times \mathcal{X} \rightarrow \mathbb{R}^{n}$ is $C^{\infty}, \mathcal{X}$ is positively invariant for $(1)$, and $f(t, 0)=0$ for all $t \geq 0$. We always assume that (1) is periodic of period $T$ in $t$, meaning there is a constant $T>0$ so that $f(t+T, x)=f(t, x)$ for all $(t, x) \in[0, \infty) \times \mathcal{X}$. We further assume that 11 is forward complete, meaning for each initial condition $x\left(t_{0}\right)=x_{0}$ with $t_{0} \geq 0$ and $x_{0} \in \mathcal{X}$, the solution $x\left(t, t_{0}, x_{0}\right)$ for the corresponding initial value problem for $(1)$ is uniquely defined on $\left[t_{0}, \infty\right)$. Set $\mathbb{N}=\{1,2,3, \ldots\}$. 
Given a $C^{\infty}$ function $V:[0, \infty) \times \mathcal{X} \rightarrow \mathbb{R}$, we use the functions

$$
a_{0}(t, x)=V(t, x), \quad \text { and } \quad a_{r}(t, x)=-\frac{\partial a_{r-1}}{\partial x}(t, x) f(t, x)-\frac{\partial a_{r-1}}{\partial t}(t, x) \forall r \in \mathbb{N} .
$$

If $V$ and $f$ are time invariant, then $a_{r}=(-1)^{r} L_{f}^{r} V$ for all $r \geq 1$, where $L_{f}^{r}$ is the usual iterated Lie derivative defined by $L_{f}^{0} V=V, L_{f} V(x)=L_{f}^{1} V(x)=\frac{\partial V}{\partial x}(x) f(x)$ and $L_{f}^{k} V=L_{f}\left(L_{f}^{k-1} V\right)$. Also, if we use $\dot{G}=(\partial G / \partial t)(t, x)+(\partial G / \partial x)(t, x) f(t, x)$ for any $C^{1}$ function $G$, then $\dot{a}_{r}=-a_{r+1}$ for all $r \geq 1$. A continuous function $k:[0, \infty) \rightarrow[0, \infty)$ is of class $\mathcal{K}_{\infty}$ (written $k \in \mathcal{K}_{\infty}$ ) provided it is zero at zero, strictly increasing and unbounded. A function $G:[0, \infty) \times \mathcal{X} \rightarrow \mathbb{R}$ is positive definite (resp., positive semi-definite) on $\mathcal{X}$ provided $G(t, 0)=0$ for all $t$ and $\inf \{G(t, x): t \geq 0\}>0$ (resp., $\geq 0$ ) for all $x \in \mathcal{X} \backslash\{0\}$. A function $G$ is negative (semi-)definite provided $-G$ is positive (semi-)definite. We use $|\cdot|$ to denote the usual Euclidean norm.

A function $V:[0, \infty) \times \mathcal{X} \rightarrow \mathbb{R}$ is called a storage function provided there are continuous positive definite functions $\alpha_{1}, \alpha_{2}: \mathcal{X} \rightarrow[0, \infty)$ such that (I) for each $i, \alpha_{i}(q) \rightarrow+\infty$ whenever $|q| \rightarrow \infty$ with $q$ remaining in $\mathcal{X}$ (i.e., $\alpha_{i}\left(q_{j}\right) \rightarrow \infty$ for each $i$ and each unbounded sequence $\left\{q_{j}\right\}$ that remains in $\mathcal{X}$ ) and (II) $\alpha_{1}(x) \leq V(t, x) \leq \alpha_{2}(x)$ for all $(t, x) \in[0, \infty) \times \mathcal{X}$. A $C^{1}$ storage function $V$ is called a nonstrict (resp., strict) Lyapunov-like function for $(1)$ provided $-a_{1}(t, x)$ is negative semi-definite (resp., negative definite). If, in addition, for each $i$ and each boundary point $\bar{q} \in \partial \mathcal{X}, \alpha_{i}(q) \rightarrow+\infty$ when $q \rightarrow \bar{q}$, then a nonstrict (resp., strict) Lyapunov-like function is called a nonstrict (resp., strict) Lyapunov function. The existence of strict Lyapunov functions is key to proving uniform global asymptotic stability (UGAS) [13].

\section{First Construction: Iterated Lie DeRivatives}

To motivate our assumptions, suppose that a given $C^{\infty}$ time invariant system $\dot{x}=f(x)$, satisfying $f(0)=0$ and evolving on $\mathbb{R}^{n}$, admits a time invariant $C^{\infty}$ nonstrict Lyapunov function $V(x)$ such that for each $q \in \mathbb{R}^{n} \backslash\{0\}$, there is an $i \in \mathbb{N}$ such that $L_{f}^{i} V(q) \neq 0$, e.g., conditions (i)-(ii) in Theorem 1 below hold, and (3) holds independently of $t$. If $L_{f} V\left(x\left(t, x_{0}\right)\right) \equiv 0$ along some trajectory 
$t \mapsto x\left(t, x_{0}\right)$ of the system, then we can differentiate repeatedly in time to get $L_{f}^{k} V\left(x\left(t, x_{0}\right)\right) \equiv 0$ for all $t \geq 0$ and all $k \in \mathbb{N}$, so $L_{f}^{k}\left(x_{0}\right)=0$ for all $k \in \mathbb{N}$. By assumption, this implies that $x_{0}=0$. Hence, UGAS follows from LaSalle invariance. However, it is not clear how to construct a global strict Lyapunov function. This motivates our (more general) hypotheses in the following theorem:

Theorem 1: Consider the periodic time varying system (1) with state space $\mathcal{X}=\mathbb{R}^{n}$ and some period $T>0$ in $t$, where $f \in C^{\infty}$. Assume that there exists a $C^{\infty}$ storage function $V:[0, \infty) \times \mathbb{R}^{n} \rightarrow$ $[0, \infty)$ having period $T$ in $t$ such that the following hold: (i) $V$ is a nonstrict Lyapunov function for (1); and (ii) there exist a constant $\tau \in(0, T]$, a constant $\ell \in \mathbb{N}$, and a positive definite continuous function $\rho$ such that for all $x \in \mathbb{R}^{n}$ and all $t \in[0, \tau]$,

$$
a_{1}(t, x)+\sum_{m=2}^{\ell} a_{m}^{2}(t, x) \geq \rho(V(t, x))
$$

Then we can explicitly determine functions $\mathcal{F}_{j}$ and $\mathcal{G}$, with $\mathcal{G}$ periodic of period $T$ in $t$, such that

$$
\begin{aligned}
& V^{\sharp}(t, x)=\sum_{j=1}^{\ell-1} \mathcal{F}_{j}(V(t, x)) A_{j}(t, x)+\mathcal{G}(t, V(t, x)), \\
& \text { where } A_{j}(t, x)=\sum_{m=1}^{j} a_{m+1}(t, x) a_{m}(t, x) \quad \forall j
\end{aligned}
$$

is a strict Lyapunov function for (1), giving UGAS of (1).

Remark 1: Theorem 1 remains true if $V$ is merely $C^{l+1}$ (instead of $C^{\infty}$ ). The assumptions of Theorem 1 are related to, but more general than, those of the strict Lyapunov function construction from [14] and different from those of [11, Corollary 2]. The assumptions of [14] are the special case of (i)-(ii) in which $f$ and $V$ are time invariant; in that case, (3) says there is a continuous positive definite function $\rho$ so that $-L_{f} V(x)+\sum_{m=2}^{\ell}\left(L_{f}^{m}(x)\right)^{2} \geq \rho(V(x))$ for all $x \in \mathbb{R}^{n}$. Our result is new, even in this special case, because the strict Lyapunov function construction in our proof of Theorem 1 is simpler than the one in [14]; see Remark 2 on $p .10$ below for details. It is important to have strict Lyapunov functions that are as simple as possible for feedback design and robustness analysis. We prove Theorem 1 in Section $\mathrm{V}$. 


\section{Second Construction: Matrosov Conditions}

To simplify the notation in our next theorem, we consider only time invariant systems

$$
\dot{x}=f(x), \quad x \in \mathcal{X}
$$

for which $\mathcal{X} \subseteq \mathbb{R}^{n}$ is positively invariant, where $f(0)=0$; see Remark 5 on $\mathrm{p} 13$ below for the generalization to (1). We use the Matrosov approach from [13] to construct global strict Lyapunov functions for (5). In addition to a nonstrict Lyapunov function, the Matrosov results from [13] require appropriate auxiliary functions, which can be difficult to find in practice. The paper [13] does not provide any general methods for constructing auxiliary functions.

The theorem we give in this section provides a new mechanism for choosing auxiliary functions. However, its most important features are that (A) it applies to systems whose state space is only a subset of $\mathbb{R}^{n}$ and (B) it may yield Lyapunov functions that are simpler than the ones from Theorem 1. and that also have desirable local properties, such as local boundedness from below by positive definite quadratic functions; see Section VII-B. For the rest of this work, we assume that all of our functions are sufficiently smooth. Our Matrosov-type assumption is:

Assumption 1: There exist a storage function $V_{1}: \mathcal{X} \rightarrow[0, \infty)$; functions $h_{1}, \ldots, h_{m}$ such that $h_{j}(0)=0$ for all $j$; everywhere positive functions $r_{1}, \ldots, r_{m}$ and $\rho$; and an integer $N>0$ for which

$$
\begin{aligned}
\nabla V_{1}(x) f(x) & \leq-r_{1}(x) h_{1}^{2}(x)-\ldots-r_{m}(x) h_{m}^{2}(x) \text { and } \\
\sum_{l=0}^{N-1} \sum_{j=1}^{m}\left[L_{f}^{l} h_{j}(x)\right]^{2} & \geq \rho\left(V_{1}(x)\right) V_{1}(x)
\end{aligned}
$$

hold for all $x \in \mathcal{X}$. Moreover, $f$ is defined on $\mathbb{R}^{n}$, and there is a function $\bar{\Gamma} \in \mathcal{K}_{\infty}$ such that

$$
|f(x)| \leq \bar{\Gamma}(|x|) \forall x \in \mathbb{R}^{n}
$$

Also, $V_{1}$ has a positive definite quadratic lower bound in some neighborhood of the origin. In Section VI, we prove: 
Theorem 2: If (5) satisfies Assumption 1, then one can determine explicit functions $k_{l}, \Omega_{l} \in$ $\mathcal{K}_{\infty} \cap C^{1}$ and an everywhere positive function $\varrho \in C^{1}$ such that

$$
S(x)=\sum_{i=1}^{N} \Omega_{i}\left(k_{i}\left(V_{1}(x)\right)+V_{i}(x)\right)
$$

with the choices

$$
V_{i}(x)=-\sum_{l=1}^{m} L_{f}^{i-2} h_{l}(x) L_{f}^{i-1} h_{l}(x), \quad i=2, \ldots, N
$$

satisfies $S(x) \geq V_{1}(x)$ and $\nabla S(x) f(x) \leq-\varrho(x) V_{1}(x)$ for all $x \in \mathcal{X}$. If, in addition, $\mathcal{X}=\mathbb{R}^{n}$, then the system (5) is GAS.

Throughout what follows, all inequalities should be understood to hold globally unless otherwise indicated, and we leave out the arguments of our functions when they are clear.

\section{Proof of Theorem 1}

1) Formula for Strict Lyapunov Function: We can assume that $\ell \geq 3$, by enlarging $\ell$ as needed without relabeling. We show that (1) admits the global strict Lyapunov function

$$
\begin{aligned}
& V^{\sharp}(t, x)=V(t, x) S_{3}(t, x)+\kappa(V(t, x)) V(t, x), \text { where } S_{3}(t, x)=S_{1}(t, x)+S_{2}(t, x), \\
& S_{1}(t, x)=\sum_{p=1}^{\ell-1} k_{p}(V(t, x)) M_{p}(t, x)+k_{0}(V(t, x)) V(t, x), \\
& S_{2}(t, x)=G(V(t, x))+\frac{1}{T}\left(\int_{t-T}^{t} \int_{s}^{t} q(r) \mathrm{d} r \mathrm{~d} s\right) k_{\ell-1}(V(t, x)) \frac{\omega(V(t, x))}{K(V(t, x))}, \text { and } \\
& M_{p}(t, x)=\sum_{m=1}^{p} a_{m+1}(t, x) a_{m}(t, x)+\int_{0}^{V(t, x)} \Gamma(r) \mathrm{d} r \text { for } p=1,2, \ldots, \ell-1 .
\end{aligned}
$$

Here $\kappa \in C^{1}$ is any strictly increasing function such that $\kappa(V(t, x)) \geq\left|S_{3}(t, x)\right|+1$ for all $(t, x) \in[0, \infty) \times \mathbb{R}^{n}, \Gamma \in C^{1}$ is any everywhere positive increasing function such that $\Gamma(V(t, x)) \geq$ $(\ell+2)\left|a_{m}(t, x)\right|+1$ for all $m \in\{1, \ldots, \ell+1\}$ and all $(t, x) \in[0, \infty) \times \mathbb{R}^{n}, \omega \in \mathcal{K}_{\infty} \cap C^{1}$ and the strictly increasing everywhere positive function $K \in C^{1}$ are such that

$$
\rho(r) \geq \frac{\omega(r)}{K(r)} \forall r \geq 0
$$


the $C^{1}$ positive definite functions $k_{1}, k_{2}, \ldots, k_{\ell-1}$ are defined by

$$
\begin{aligned}
& k_{\ell-1}(v)=\omega^{2^{\ell-1}}(v) \text { and } k_{p}(v)=k_{\ell-1}(v) \Omega^{1-2^{\ell-p-1}}(v) \text { for } p=1,2, \ldots, \ell-2, \\
& \text { where } \Omega(v)=\frac{2 \tau \omega(v)}{3 T(\ell-2) \Gamma^{2}(v) K(v)},
\end{aligned}
$$

$k_{0}$ is any $C^{1}$ increasing function such that

$$
k_{0}(V(t, x))+k_{0}^{\prime}(V(t, x)) V(t, x) \geq \sum_{p=1}^{\ell-1}\left|k_{p}^{\prime}(V(t, x))\right|\left|M_{p}(t, x)\right|+1
$$

$q: \mathbb{R} \rightarrow[0,1]$ is any continuous function with period $T$ that satisfies $q(t)=0$ for all $t \in[\tau, T]$ and $q(t)=1$ for all $t \in\left[\frac{\tau}{3}, \frac{2 \tau}{3}\right]$, and $G$ is any $C^{1}$ function such that

$$
G^{\prime}(v) \geq T\left|k_{\ell-1}(v) \frac{\omega^{\prime}(v) K(v)-\omega(v) K^{\prime}(v)}{K^{2}(v)}+k_{\ell-1}^{\prime}(v) \frac{\omega(v)}{K(v)}\right|
$$

for all $v \geq 0$. The functions $\omega$ and $K$ can be obtained using Lemma A.1 below, $\Gamma$ can be obtained by majorizing $s \mapsto 1+\max \left\{(\ell+2)\left|a_{m}(t, x)\right|: t \geq 0, x \in \mathcal{X}, m \in\{1,2, \ldots, \ell+1\}, V(t, x) \leq s\right\}$ by a $C^{1}$ function, and $k_{0}$ can be obtained because each $M_{p}$ is periodic in $t$ and because $V$ is a storage function that is also periodic in $t$. The theorem will then follow by collecting the functions involving $V$ to produce the formula (4) for $V^{\sharp}$.

2) Stability Analysis: To show that (10) is a strict Lyapunov function for (1), we use the everywhere nonnegative functions $N_{j}(t, x)=\sum_{m=2}^{j+1} a_{m}^{2}(t, x)+a_{1}(t, x)$ for $1 \leq j \leq \ell-1$, which have period $T$ in $t$. By (3), (11), and the nonnegativity of $N_{\ell-1}$,

$$
N_{\ell-1}(t, x) \geq q(t) \frac{\omega(V(t, x))}{K(V(t, x))}
$$

for all $(t, x) \in[0, \infty) \times \mathbb{R}^{n}$. Since $a_{1}=-\dot{V} \geq 0$, our choice of $\Gamma$ and 10$)$ give

$$
\begin{aligned}
\dot{M}_{1} & =\dot{a}_{2} a_{1}-a_{2}^{2}-\Gamma(V) a_{1} \leq-N_{1}, \text { and } \\
\dot{M}_{j} & \leq-\sum_{m=1}^{j} a_{m+1}^{2}+\sum_{m=2}^{j}\left|a_{m+2}\right|\left|a_{m}\right|+\left|a_{3}\right| a_{1}-\Gamma(V) a_{1} \\
& \leq-\sum_{m=1}^{j} a_{m+1}^{2}+\sum_{m=2}^{j}\left|a_{m+2}\right|\left|a_{m}\right|+\left|a_{3}\right| a_{1}-\left[(\ell+2)\left|a_{3}\right|+1\right] a_{1}
\end{aligned}
$$


for each $j \in\{2, \ldots, \ell-1\}$, since $\dot{a}_{i}=-a_{i+1}$ for all $i$. Recalling our choice of $\Gamma$ gives

$$
\dot{M}_{j} \leq-\sum_{m=1}^{j} a_{m+1}^{2}+\frac{\Gamma(V)}{\ell+2} \sum_{m=2}^{j}\left|a_{m}\right|-\left[(\ell+1)\left|a_{3}\right|+1\right] a_{1} \quad \forall j \in\{2, \ldots, \ell-1\} .
$$

It follows from the Cauchy Inequality that for all $j \in\{2, \ldots, \ell-1\}$,

$$
\begin{aligned}
\dot{M}_{j} & \leq-\sum_{m=1}^{j} a_{m+1}^{2}+\Gamma(V) \sqrt{\sum_{m=2}^{j} a_{m}^{2}}-\left[(\ell+1)\left|a_{3}\right|+1\right] a_{1} \\
& \leq-N_{j}+\Gamma(V) \sqrt{N_{j-1}},
\end{aligned}
$$

since $a_{1}=-\dot{V} \geq 0$ everywhere. Also,

$$
\begin{aligned}
\dot{S}_{1} & =\sum_{p=1}^{\ell-1} k_{p}(V) \dot{M}_{p}+\left[\sum_{p=1}^{\ell-1} k_{p}^{\prime}(V) M_{p}\right] \dot{V}+\left[k_{0}(V)+k_{0}^{\prime}(V) V\right] \dot{V} \\
& \leq \sum_{p=1}^{\ell-1} k_{p}(V) \dot{M}_{p}+\left[\sum_{p=1}^{\ell-1}\left|k_{p}^{\prime}(V)\right|\left|M_{p}\right|\right][-\dot{V}]+\left[k_{0}(V)+k_{0}^{\prime}(V) V\right] \dot{V} \\
& \leq \sum_{p=1}^{\ell-1} k_{p}(V) \dot{M}_{p},
\end{aligned}
$$

using 13 and the fact that $\dot{V}$ is nonpositive. Using 16 - 17 , we deduce that

$$
\begin{aligned}
\dot{S}_{1} & \leq-k_{1}(V) N_{1}+\sum_{p=2}^{\ell-1} k_{p}(V)\left[-N_{p}+\Gamma(V) \sqrt{N_{p-1}}\right] \\
& \leq-\sum_{p=1}^{\ell-1} k_{p}(V) N_{p}+\sum_{p=2}^{\ell-1} k_{p}(V) \Gamma(V) \sqrt{N_{p-1}} .
\end{aligned}
$$

It follows from (15) that

$$
\dot{S}_{1} \leq-k_{\ell-1}(V) q(t) \frac{\omega(V)}{K(V)}-\sum_{p=1}^{\ell-2} k_{p}(V) N_{p}+\sum_{p=1}^{\ell-2} k_{p+1}(V) \Gamma(V) \sqrt{N_{p}} .
$$

Since $\int_{t-T}^{t} \int_{s}^{t} q(r) \mathrm{d} r \mathrm{~d} s \leq T^{2}$ and $\frac{d}{d t} \int_{t-T}^{t} \int_{s}^{t} q(r) \mathrm{d} r \mathrm{~d} s=T q(t)-\int_{t-T}^{t} q(r) \mathrm{d} r, 14$ gives

$$
\dot{S}_{2} \leq k_{\ell-1}(V) q(t) \frac{\omega(V)}{K(V)}-\frac{1}{T}\left(\int_{t-T}^{t} q(r) \mathrm{d} r\right) k_{\ell-1}(V) \frac{\omega(V)}{K(V)} .
$$

Since $\int_{t-T}^{t} q(r) \mathrm{d} r \geq \frac{\tau}{3}$ for all $t \in \mathbb{R}, \sqrt{19}$ and 20 give

$$
\begin{aligned}
\dot{S}_{3} & \leq-\frac{1}{T}\left(\int_{t-T}^{t} q(r) \mathrm{d} r\right) k_{\ell-1}(V) \frac{\omega(V)}{K(V)}-\sum_{p=1}^{\ell-2} k_{p}(V) N_{p}+\sum_{p=1}^{\ell-2} k_{p+1}(V) \Gamma(V) \sqrt{N_{p}} \\
& \leq-\frac{\tau}{3 T} k_{\ell-1}(V) \frac{\omega(V)}{K(V)}-\sum_{p=1}^{\ell-2} k_{p}(V) N_{p}+\sum_{p=1}^{\ell-2} k_{p+1}(V) \Gamma(V) \sqrt{N_{p}} .
\end{aligned}
$$


From the triangular inequality $c_{1} c_{2} \leq c_{1}^{2}+\frac{1}{4} c_{2}^{2}$ for nonnegative values $c_{1}$ and $c_{2}$, we deduce that

$$
k_{p+1}(V) \Gamma(V) \sqrt{N_{p}}=\left\{\sqrt{k_{p}(V) N_{p}}\right\}\left\{\frac{\Gamma(V) k_{p+1}(V)}{\sqrt{k_{p}(V)}}\right\} \leq k_{p}(V) N_{p}+\frac{\Gamma^{2}(V) k_{p+1}^{2}(V)}{4 k_{p}(V)}
$$

for $p=1,2, \ldots, l-2$ when $V \neq 0$. Summing in 22) over $p$ and substituting into (21) gives

$$
\dot{S}_{3} \leq-\frac{\tau}{3 T} k_{\ell-1}(V) \frac{\omega(V)}{K(V)}+\sum_{p=1}^{\ell-2} \frac{\Gamma^{2}(V) k_{p+1}^{2}(V)}{4 k_{p}(V)}
$$

when $V \neq 0$. By our choices 2 of the $k_{p}$ 's and $\Omega$, 23 gives

$$
\begin{aligned}
\dot{S}_{3} & \leq-\frac{\tau}{3 T} k_{\ell-1}(V) \frac{\omega(V)}{K(V)}+\sum_{p=1}^{\ell-2} \frac{\Gamma^{2}(V) k_{\ell-1}^{2}(V) \Omega^{2\left(1-2^{\ell-p-2}\right.}(V)}{4 k_{\ell-1}(V) \Omega^{1-2^{\ell-p-1}}(V)} \\
& \leq-\frac{\tau}{3 T} k_{\ell-1}(V) \frac{\omega(V)}{K(V)}+(\ell-2) \frac{\Gamma^{2}(V) k_{\ell-1}(V) \Omega(V)}{4} \leq-\frac{\tau}{6 T} k_{\ell-1}(V) \frac{\omega(V)}{K(V)}, \quad V \neq 0 .
\end{aligned}
$$

Hence, $\dot{S}_{3}$ is negative definite. However, $S_{3}$ is not necessarily positive definite and radially unbounded, and so may not be a strict Lyapunov function.

To check that $V^{\sharp}$ from 10 is a strict Lyapunov function, first note that it is positive definite and radially unbounded because $V^{\sharp}(t, x) \geq V(t, x)$, by our choice of $\kappa$. Also, since $\dot{V} \leq 0$ everywhere,

$$
\dot{V}^{\sharp}=V \dot{S}_{3}+\dot{V} S_{3}+\left[\kappa^{\prime}(V) V+\kappa(V)\right] \dot{V} \leq-\frac{\tau}{6 T} k_{\ell-1}(V) \frac{\omega(V)}{K(V)} V,
$$

which is the desired strict Lyapunov function decay condition. This proves Theorem 1 .

Remark 2: For the special case of time-invariant systems, the assumptions from Theorem 1 agree with the assumptions from the strict Lyapunov function construction in [14, Theorem 3.1]. However, our proof of Theorem 1 is simpler than the arguments from [14]. The construction in [14, Theorem 3.1] requires a non-increasing function $\lambda:[0, \infty) \rightarrow(0, \infty)$ such that the function

$$
U(x)=V(x)\left[1+V(x)-\sum_{i=1}^{\ell-1} L_{f_{\lambda}}^{i} V(x) \cdot\left(L_{f_{\lambda}}^{i+1} V(x)\right)^{3^{i}}\right]
$$

is a strict Lyapunov function for the system, where $f_{\lambda}(x):=\lambda(V(x)) f(x)$. There is no analog of $\lambda$ in our simpler proof of Theorem 1 . 


\section{Proof of TheOREM 2}

In the following proof, we omit the dependencies of the functions on $x$ when they are clear from the context. To simplify our notation, we introduce the functions

$$
\begin{aligned}
& \mathcal{N}_{1}(x)=R(x) \sum_{l=1}^{m} h_{l}^{2}(x) \text { and } \mathcal{N}_{i}(x)=\sum_{l=1}^{m}\left[L_{f}^{i-1} h_{l}(x)\right]^{2} \forall i \geq 2, \\
& \text { where } R(x)=\frac{\prod_{i=1}^{m} r_{i}(x)}{\prod_{i=1}^{m}\left[r_{i}(x)+1\right]}
\end{aligned}
$$

Since $R$ is everywhere positive and satisfies $R(x) \leq r_{i}(x)$ for all $x \in \mathbb{R}^{n}$ and all $i \in\{1, \ldots, m\}$, (6) and our choices (9) of the $V_{i}$ 's give

$$
\begin{aligned}
& \nabla V_{1}(x) f(x) \leq-\mathcal{N}_{1} \forall x \in \mathcal{X}, \text { and } \\
& \nabla V_{i}(x) f(x)=-\sum_{l=1}^{m}\left[L_{f}^{i-1} h_{l}\right]^{2}-\sum_{l=1}^{m} L_{f}^{i-2} h_{l} L_{f}^{i} h_{l} \leq-\mathcal{N}_{i}+\sum_{l=1}^{m}\left|L_{f}^{i-2} h_{l}\right|\left|L_{f}^{i} h_{l}\right|
\end{aligned}
$$

for $i=2, \ldots, N$ and all $x \in \mathcal{X}$. In particular, we have

$$
\begin{aligned}
& \nabla V_{2}(x) f(x) \leq-\mathcal{N}_{2}(x)+\sqrt{\mathcal{N}_{1}(x)} \sum_{l=1}^{m} \frac{\left|L_{f}^{2} h_{l}(x)\right|}{\sqrt{R(x)}}, \text { and } \\
& \nabla V_{i}(x) f(x) \leq-\mathcal{N}_{i}(x)+\sqrt{\mathcal{N}_{i-1}(x)} \sum_{l=1}^{m}\left|L_{f}^{i} h_{l}(x)\right| \text { for } i=3,4, \ldots, N
\end{aligned}
$$

Our assumptions on $f, V_{1}$, and the $h_{i}$ 's allow us to determine a function $\underline{\alpha} \in \mathcal{K}_{\infty}$ such that $V_{1}(x) \geq \underline{\alpha}(|x|)$ for all $x \in \mathcal{X}$, and a continuous everywhere positive function $\phi_{1}$ such that

$$
\sum_{l=1}^{m} \frac{\left|L_{f}^{2} h_{l}(x)\right|}{\sqrt{R(x)}} \leq \phi_{1}\left(V_{1}(x)\right) \sqrt{V_{1}(x)} \quad \text { and } \quad \sum_{l=1}^{m}\left|L_{f}^{i} h_{l}(x)\right| \leq \phi_{1}\left(V_{1}(x)\right) \sqrt{V_{1}(x)}
$$

for $i=3, \ldots, N$ and all $x \in \mathcal{X}$; see Appendix A.2 below. It follows that for all $i \geq 2$ and $x \in \mathcal{X}$,

$$
\nabla V_{i}(x) f(x) \leq-\mathcal{N}_{i}(x)+\phi_{1}\left(V_{1}(x)\right) \sqrt{\mathcal{N}_{i-1}(x)} \sqrt{V_{1}(x)}
$$

Arguing as we did to get 27) gives an increasing continuous everywhere nonnegative function $p_{1} \in C^{1}$ such that $\left|V_{i}(x)\right| \leq p_{1}\left(V_{1}(x)\right) V_{1}(x)$ for $i=1, \ldots, N$ for all $x \in \mathcal{X}$ (by first finding an increasing positive function $\tilde{\alpha}$ so that $\left|V_{i}(x)\right| \leq \tilde{\alpha}(|x|)|x|^{2}$ for all $i \geq 2$ and all $x$ near 0 , using the 
fact that $h_{r}(0)=0$ for all $r$ to handle the $i=2$ case). Finally, we can find a decreasing everywhere positive function $\underline{\rho}$ so that $R(x) \geq \underline{\rho}(\underline{\alpha}(|x|)) \geq \underline{\rho}\left(V_{1}(x)\right)$ on $\mathcal{X}$, and then a continuous everywhere positive function $\tilde{\rho}$ so that

$$
\sum_{i=1}^{N} \mathcal{N}_{i}(x) \geq \tilde{\rho}\left(V_{1}(x)\right) V_{1}(x)
$$

on $\mathcal{X}$, by $(6)$. Hence, the assumptions of [13, Theorem 1] hold with $a_{i} \equiv \frac{1}{2}$, so [13, Theorem 1] constructs the necessary strict Lyapunov-like function.

Remark 3: As we noted above, the results of [13] do not provide general methods for building the auxiliary functions from the Matrosov conditions. Therefore, the novelty of the preceding proof lies in its general procedure for producing the functions $V_{i}$ that satisfy the Matrosov conditions. On the other hand, given the functions $V_{i}, \phi_{1}, p_{1}$, and $\tilde{\rho}$ we constructed above, the results of [13] readily produce the desired strict Lyapunov function $S$. The strict Lyapunov function takes the form

$$
S(x)=\Omega_{1}\left(2 V_{1}(x)\right)+\sum_{i=2}^{N} \Omega_{i}\left(U_{i}(x)\right)
$$

where

$$
U_{i}(x)=V_{i}(x)+V_{1}(x)\left[1+p_{1}\left(V_{1}(x)\right)\right]
$$

for all $i \geq 2$, and where the functions $\Omega_{i} \in \mathcal{K}_{\infty} \cap C^{1}$ are recursively chosen as follows. We take $\Omega_{N}(r)=r$, and then choose $\Omega_{i}$ for $i=1,2, \ldots, N-1$ to satisfy

$$
\Omega_{i}^{\prime}\left(U_{i}\right) \geq(N-1)^{2} \frac{8 \phi_{1}^{2}\left(V_{1}\right)}{\tilde{\rho}\left(V_{1}\right)} \sum_{r=1+i}^{N} \Omega_{r}^{\prime}\left(U_{r}\right)^{2}
$$

with $\Omega_{i}^{\prime}:[0, \infty) \rightarrow[1, \infty)$ continuous and increasing for each $i$. The formula 29 is the special case of the construction from [13, Theorem 1] when all of the exponents $a_{i}$ in [13] are $\frac{1}{2}$.

Remark 4: A standard "smoothing of the corners" argument that is analogous to the one used to prove [4, Lemma 2.5] allows us to majorize (resp., minorize) any continuous increasing (resp., decreasing) function $\phi:[0, \infty) \rightarrow(0, \infty)$ by a $C^{\infty}$ everywhere positive increasing (resp., decreasing) 
function. Therefore, when $f$ and $V_{1}$ are both $C^{\infty}$, we can use the argument from [13, Theorem 1] in conjunction with smoothing of the corners to guarantee that the functions $\Omega_{i}$ are $C^{\infty}$, hence a $C^{\infty}$ strict Lyapunov function that is consistent with the known converse Lyapunov function theory.

Remark 5: We can prove an analog of Theorem 2 for (1), under a periodic time varying version of Assumption 11. The periodic time varying analog of Assumption 1 is obtained by (A) replacing the arguments of $f$ and the $V_{i}$ 's by $(t, x)$ and (B) replacing $\nabla V_{i}(x) f(x)$ with

$$
\frac{\partial V_{i}}{\partial t}(t, x)+\frac{\partial V_{i}}{\partial x}(t, x) f(t, x)
$$

The proof is then as before, using the periodic time varying extension in [13, Section IV].

\section{ILLUSTRATIONS}

\section{A. Periodic Time Varying System}

To illustrate Theorem 1 in a reasonably simple way, we take the periodic time varying system

$$
\left\{\begin{array}{l}
\dot{x}_{1}=\cos (t) x_{2} \\
\dot{x}_{2}=-\cos (t) x_{1}-x_{2} .
\end{array}\right.
$$

Along the trajectories of 32 , the nonstrict Lyapunov function $V(x)=\frac{1}{2}|x|^{2}$ gives $\dot{V}=-x_{2}^{2}$. Using the notation from Theorem 1 , we get $a_{1}(t, x)=x_{2}^{2}$, so $\dot{a}_{1}=-2 \cos (t) x_{1} x_{2}-2 x_{2}^{2}$. This gives $a_{2}(t, x)=2 \cos (t) x_{1} x_{2}+2 x_{2}^{2}$, hence

$$
\dot{a}_{2}=-2 \cos ^{2}(t) x_{1}^{2}-2\left[(3 \cos (t)+\sin (t)) x_{1}+\left(1+\sin ^{2}(t)\right) x_{2}\right] x_{2}
$$

Therefore, $a_{3}(t, x)=2 \cos ^{2}(t) x_{1}^{2}+2\left[(3 \cos (t)+\sin (t)) x_{1}+\left(1+\sin ^{2}(t)\right) x_{2}\right] x_{2}$. Using the relations $2 p^{2}+2 q^{2} \geq(p+q)^{2} \geq \frac{p^{2}}{10}-\frac{q^{2}}{9}$ for any $p \geq 0$ and $q \geq 0$ gives

$$
\begin{aligned}
a_{3}^{2}(t, x) & \geq \frac{2}{5} \cos ^{4}(t) x_{1}^{4}-\frac{4}{9}\left[\{3 \cos (t)+\sin (t)\} x_{1}+\left\{1+\sin ^{2}(t)\right\} x_{2}\right]^{2} x_{2}^{2} \\
& \geq \frac{2}{5} \cos ^{4}(t) x_{1}^{4}-\frac{16}{9}\left[2\left|x_{1}\right|+\left|x_{2}\right|\right]^{2} x_{2}^{2} \geq \frac{2}{5} \cos ^{4}(t) x_{1}^{4}-29 V(x) a_{1}(t, x)
\end{aligned}
$$


hence

$$
\frac{1}{40(V(x)+1)} a_{3}^{2}(t, x) \geq \frac{1}{100(V(x)+1)} \cos ^{4}(t) x_{1}^{4}-\frac{3}{4} a_{1}(t, x)
$$

Noticing that $\frac{1}{40(V(x)+1)}<1$ and $V^{2}(x) \leq \frac{1}{2}\left(x_{1}^{4}+x_{2}^{4}\right)$, we deduce from $34-35$ that

$$
\begin{aligned}
a_{1}(t, x)+a_{2}^{2}(t, x)+a_{3}^{2}(t, x) & \geq \frac{\cos ^{4}(t)}{100(V(x)+1)} x_{1}^{4}+\frac{1}{4} a_{1}(t, x) \geq \frac{\cos ^{4}(t)}{100(V(x)+1)} x_{1}^{4}+\frac{1}{8(V(x)+1)} x_{2}^{4} \\
& \geq \frac{\cos ^{4}(t)}{100(V(x)+1)}\left[x_{1}^{4}+x_{2}^{4}\right] \geq \frac{4 \cos ^{4}(t)}{200(V(x)+1)} V^{2}(x)
\end{aligned}
$$

Since $4 \cos ^{4}(t) \geq 1$ on $[0, \pi / 4]$, we can satisfy the assumptions of Theorem 1 with $V(x)=\frac{1}{2}|x|^{2}$, $\ell=3, T=2 \pi, \tau=\frac{\pi}{4}$, and $\rho(r)=r^{2} /\{200(r+1)\}$. See Appendix A.3 for the construction of the strict Lyapunov function $V^{\sharp}$ that follows the proof of Theorem 1 .

\section{B. Lotka-Volterra Example}

We illustrate Theorem 2 using the celebrated Lotka-Volterra Predator-Prey system

$$
\left\{\begin{array}{l}
\dot{\chi}=\gamma \chi\left(1-\frac{\chi}{L}\right)-a \chi \zeta \\
\dot{\zeta}=\beta \chi \zeta-\Delta \zeta
\end{array}\right.
$$

with positive constants $a, \beta \gamma, \Delta$, and $L$. System 36 is a simple model of one predator feeding on one prey. The population of the predator is $\zeta, \chi$ is the population of the prey, and the constants are related to the birth and death rates; see [6], [10] for an extensive analysis of this model and generalizations to several predators. We assume that the population levels are positive. While there are many Lyapunov constructions for Lotka-Volterra models available (based on computing the LaSalle invariant set), to the best of our knowledge, the result to follow is original and significant because we provide a global strict Lyapunov function.

1) Global Strict Lyapunov Function Construction: The time scaling, change of coordinates, and constants

$$
x(t)=\frac{1}{L} \chi\left(\frac{t}{\gamma}\right), \quad y(t)=\frac{a}{\beta L} \zeta\left(\frac{t}{\gamma}\right), \quad \alpha=\frac{\beta L}{\gamma} \text { and } d=\frac{\Delta}{\gamma}
$$


give the simpler system

$$
\left\{\begin{array}{l}
\dot{x}=x(1-x)-\alpha x y \\
\dot{y}=\alpha x y-d y
\end{array}\right.
$$

We assume that we have imposed assumptions on the parameters such that $\alpha>d$. Let

$$
x_{*}=\frac{d}{\alpha} \quad \text { and } \quad y_{*}=\frac{1}{\alpha}-\frac{d}{\alpha^{2}} .
$$

Then $x_{*} \in(0,1)$ and $y_{*}>0$. Also, the new variables $\tilde{x}=x-x_{*}$ and $\tilde{y}=y-y_{*}$ have the dynamics

$$
\left\{\begin{aligned}
\dot{\tilde{x}} & =-[\tilde{x}+\alpha \tilde{y}]\left(\tilde{x}+x_{*}\right) \\
\dot{\tilde{y}} & =\alpha \tilde{x}\left(\tilde{y}+y_{*}\right)
\end{aligned}\right.
$$

with state space $\mathcal{X}=\left(-x_{*},+\infty\right) \times\left(-y_{*},+\infty\right)$. We do our Lyapunov function construction for (40), so we set

$$
f(\tilde{x}, \tilde{y})=\left(\begin{array}{c}
-[\tilde{x}+\alpha \tilde{y}]\left(\tilde{x}+x_{*}\right) \\
\alpha \tilde{x}\left(\tilde{y}+y_{*}\right)
\end{array}\right)
$$

We verify Assumption 1 with

$$
\begin{aligned}
& m=1, \quad N=2, \quad r_{1} \equiv 1, \quad h_{1}(\tilde{x}, \tilde{y})=\tilde{x}, \text { and } \\
& V_{1}(\tilde{x}, \tilde{y})=\tilde{x}-x_{*} \ln \left(1+\frac{\tilde{x}}{x_{*}}\right)+\tilde{y}-y_{*} \ln \left(1+\frac{\tilde{y}}{y_{*}}\right) .
\end{aligned}
$$

One easily checks that $V_{1}$ is a (time invariant) storage function. Along the trajectories of (40),

$$
\begin{aligned}
\dot{V}_{1} & =-\frac{\tilde{x}}{x_{*}+\tilde{x}}[\tilde{x}+\alpha \tilde{y}]\left(\tilde{x}+x_{*}\right)+\frac{\alpha \tilde{y}}{y_{*}+\tilde{y}} \tilde{x}\left(\tilde{y}+y_{*}\right) \\
& =-\tilde{x}[\tilde{x}+\alpha \tilde{y}]+\alpha \tilde{y} \tilde{x}=-\tilde{x}^{2} .
\end{aligned}
$$

Also, $L_{f} h_{1}(\tilde{x}, \tilde{y})=-[\tilde{x}+\alpha \tilde{y}]\left(\tilde{x}+x_{*}\right)$. One can check that $V_{1}$ has a positive definite quadratic lower bound near the origin; see Appendix A.4. A simple argument provides a positive constant $\underline{d}$ such that

$$
\frac{1}{2} h_{1}^{2}(\tilde{x}, \tilde{y})+\left[L_{f} h_{1}(\tilde{x}, \tilde{y})\right]^{2} \geq \underline{d} \frac{V_{1}(\tilde{x}, \tilde{y})}{1+V_{1}^{2}(\tilde{x}, \tilde{y})}
$$


on $\mathcal{X}$; see Appendix $\mathrm{A} .5$ below. Hence, Assumption 1 holds with

$$
\rho(r)=\frac{\underline{d}}{1+r^{2}}
$$

so Theorem 2 constructs the necessary global strict Lyapunov function for (40).

We construct the strict Lyapunov function from Theorem 2. We use the notation from the proof of Theorem 2, so $\mathcal{N}_{1}(\tilde{x}, \tilde{y})=\frac{1}{2} h_{1}(\tilde{x}, \tilde{y})^{2}$ and $\mathcal{N}_{2}(\tilde{x}, \tilde{y})=\left[L_{f} h_{1}(\tilde{x}, \tilde{y})\right]^{2}$. Notice that

$$
L_{f}^{2} h_{1}(\tilde{x}, \tilde{y})=-\left(x_{*}+2 \tilde{x}+\alpha \tilde{y}\right) \dot{\tilde{x}}-\left(x_{*}+\tilde{x}\right) \alpha \dot{\tilde{y}}
$$

Therefore,

$$
\begin{aligned}
\left|L_{f}^{2} h_{1}(\tilde{x}, \tilde{y})\right| \leq & \left(2+\alpha+x_{*}\right)^{3}(1+|\tilde{x}|+|\tilde{y}|)^{2}(|\tilde{x}|+|\tilde{y}|) \\
& +\alpha^{2}\left(1+x_{*}+y_{*}\right)^{2}(1+|\tilde{x}|)(1+|\tilde{y}|)|\tilde{x}| .
\end{aligned}
$$

On the other hand, Appendix A.4 below applied with $A=\tilde{x} / x_{*}$ gives

$$
\left|\frac{\tilde{x}}{x_{*}}\right| \leq 2\left\{\frac{V_{1}}{x_{*}}+\left[\frac{V_{1}}{x_{*}}\right]^{2}\right\}^{1 / 2} \leq 2\left[\max \left\{\frac{1}{x_{*}}, \frac{1}{x_{*}^{2}}\right\}\left\{V_{1}+V_{1}^{2}\right\}\right]^{1 / 2}
$$

and similarly for $y$, so

$$
\max \{|\tilde{x}|,|\tilde{y}|\} \leq J\left(V_{1}\right) \sqrt{V_{1}}, \text { where } J\left(V_{1}\right)=2\left(1+x_{*}+y_{*}\right) \sqrt{V_{1}+1}
$$

Combining (47) and (49) gives

$$
\begin{aligned}
\left|L_{f}^{2} h_{1}(\tilde{x}, \tilde{y})\right| \leq & \left\{2 J\left(V_{1}\right)\left(2+\alpha+x_{*}\right)^{3}\left[1+2 J\left(V_{1}\right) \sqrt{V_{1}}\right]^{2}\right. \\
& \left.+\alpha^{2}\left(1+x_{*}+y_{*}\right)^{2} J\left(V_{1}\right)\left(1+J\left(V_{1}\right) \sqrt{V_{1}}\right)^{2}\right\} \sqrt{V_{1}}
\end{aligned}
$$

Therefore, we can satisfy 27)- 28) with $\phi_{1}(r)=4\left(1+\alpha^{2}\right)\left(3+\alpha+y_{*}\right)^{3}(1+2 J(r) \sqrt{r})^{2} J(r)$.

Since $V_{2}(\tilde{x}, \tilde{y})=\tilde{x}[\tilde{x}+\alpha \tilde{y}]\left(\tilde{x}+x_{*}\right)$, we easily get

$$
\left|V_{2}(\tilde{x}, \tilde{y})\right| \leq 2\left(x_{*}+1\right)(1+\alpha)\left[\tilde{y}^{4}+|\tilde{x}|^{3}+\tilde{x}^{2}+\tilde{y}^{2}\right]
$$


Combining (49) and (51) and setting $\bar{d}=1+x_{*}+y_{*}$, simple algebra gives

$$
\left|V_{2}(\tilde{x}, \tilde{y})\right| \leq 4\left(x_{*}+1\right)(1+\alpha) \sum_{i=2}^{4}\left\{2 \bar{d} \sqrt{V_{1}+V_{1}^{2}}\right\}^{i} \leq p_{1}\left(V_{1}(\tilde{x}, \tilde{y})\right) V_{1}(\tilde{x}, \tilde{y})
$$

where $p_{1}(r)=640\left(x_{*}+1\right)(\alpha+1) \bar{d}^{4}(1+r)^{3}$. Since we also have

$$
\sum_{i=1}^{2} \mathcal{N}_{i}(\tilde{x}, \tilde{y}) \geq \tilde{\rho}\left(V_{1}(\tilde{x}, \tilde{y})\right) V_{1}(\tilde{x}, \tilde{y}), \text { where } \tilde{\rho}(r)=\frac{\underline{d}}{1+r^{2}}
$$

and since

$$
U_{2}(\tilde{x}, \tilde{y})=V_{2}(\tilde{x}, \tilde{y})+\left[p_{1}\left(V_{1}(\tilde{x}, \tilde{y})\right)+1\right] V_{1}(\tilde{x}, \tilde{y}) \geq V_{1}(\tilde{x}, \tilde{y})
$$

everywhere, it follows from (29)-(31) that the desired strict Lyapunov-like function we get is

$$
S(\tilde{x}, \tilde{y})=V_{2}(\tilde{x}, \tilde{y})+\left[p_{1}\left(V_{1}(\tilde{x}, \tilde{y})\right)+1\right] V_{1}(\tilde{x}, \tilde{y})+\frac{8}{\underline{d}} \int_{0}^{2 V_{1}(\tilde{x}, \tilde{y})}\left(1+r^{2}\right) \phi_{1}^{2}(r) \mathrm{d} r .
$$

Moreover, $S$ is a strict Lyapunov function because $V_{1}(\tilde{x}, \tilde{y})$ goes to infinity when $\tilde{x}$ goes to $-x_{*}$ or $+\infty$, or when $\tilde{y}$ goes to $-y_{*}$ or $+\infty$.

Remark 6: Due to its restricted state space, it is not possible to apply Theorem 1 to the LotkaVolterra example directly. However, a change of variables that transforms the state space to all of Euclidean space makes it possible to apply to Theorem 1. See Appendix A.6 below for details.

2) ISS and iISS: We can use our strict Lyapunov function constructions to quantify the effects of uncertainty in the Lotka-Volterra dynamics. We illustrate this by showing that the dynamics are ISS with respect to additive uncertainty in the death rate $\Delta$ for the predator. Using the coordinate change and constants (37), this means that we replace the constant $d>0$ with $d+\mathbf{u}$ in the dynamics (38), where $\mathbf{u}:[0, \infty) \rightarrow \mathbb{R}$ is a measurable essentially bounded uncertainty, and where $d$ now represents the nominal (or estimated) value of the parameter. Later, we impose a bound on the allowable values for $|\mathbf{u}|_{\infty}$. We continue to use $d$ in the formulas 39 for $x_{*}$ and $y_{*}$; we do not introduce uncertainty in the equilibrium values. Set $\delta \mathcal{B}_{r}=\left\{x \in \mathbb{R}^{r}:|x| \leq \delta\right\}$ for any constants 
$r \in \mathbb{N}$ and $\delta>0$.

We first define an appropriately restricted state space. Along the trajectories of (38), with $d$ replaced by $d+\mathbf{u}$, we have

$$
\dot{x}+\dot{y}=x(1-x)-(d+\mathbf{u}) y
$$

Hence, if $|\mathbf{u}|_{\infty} \leq d / 2$ with $(x, y) \in(0, \infty)^{2}$, then $\dot{x}+\dot{y}<0$ when $x+y>1+\frac{2}{d}$ (by separately considering the cases $x>1$ and $x \leq 1$ ). Therefore, we restrict to disturbances satisfying $|\mathbf{u}|_{\infty} \leq d / 2$ and the forward invariant set

$$
\mathcal{S}=\left\{(x, y) \in(0, \infty)^{2}: x+y \leq \mathcal{B}\right\}, \text { where } \mathcal{B}=1+\frac{2}{d}+y_{*},
$$

where we added $y_{*}$ to ensure that $\left(x_{*}, y_{*}\right) \in S$. The corresponding perturbed error dynamics

$$
\left\{\begin{aligned}
\dot{\tilde{x}} & =-[\tilde{x}+\alpha \tilde{y}]\left(\tilde{x}+x_{*}\right) \\
\dot{\tilde{y}} & =\alpha \tilde{x}\left(\tilde{y}+y_{*}\right)-\mathbf{u} y
\end{aligned}\right.
$$

has the state space $\mathcal{X}^{b}=\{(\tilde{x}, \tilde{y}):(x, y) \in \mathcal{S}\}$ and a control set $U$ we will specify. Our strategy is to build an appropriate strict Lyapunov function for $(54)$ for the special case where $\mathbf{u} \equiv 0$ (i.e., (40), which we then use to prove ISS of (54) with respect to the uncertainty $\mathbf{u}$.

To account for the restricted state space of the system, we use the following definitions. Given an open subset $\mathcal{D}$ of a Euclidean space that contains the origin, we say that a positive definite function $\bar{\alpha}: \mathcal{D} \rightarrow[0, \infty)$ is a modulus with respect to $\mathcal{D}$ provided $\bar{\alpha}(p) \rightarrow \infty$ as $|p| \rightarrow \infty$ or as $\operatorname{dist}(p, \partial \mathcal{D}) \rightarrow 0$ (with $p$ remaining in $\mathcal{D}$ ). We say that 54 is ISS with respect to $\mathbf{u}$ provided there exist functions $\beta \in \mathcal{K} \mathcal{L}$ and $\gamma \in \mathcal{K}_{\infty}$, and a modulus with respect to $\left(-x_{*}, \infty\right) \times\left(-y_{*}, \infty\right)$, such that for each disturbance $\mathbf{u}:[0, \infty) \rightarrow U$ and each trajectory $(\tilde{x}, \tilde{y}):[0, \infty) \rightarrow \mathcal{X}^{b}$ of 54 corresponding to $\mathbf{u}$, we have

$$
|(\tilde{x}, \tilde{y})(t)| \leq \beta(\bar{\alpha}((\tilde{x}, \tilde{y})(0)), t)+\gamma\left(|\mathbf{u}|_{\infty}\right) \quad \forall t \geq 0
$$


To simplify the statements of our results, we use the constants

$$
\begin{aligned}
& K_{0}=2\left[\frac{(3+\alpha)^{2}}{2}+\alpha^{2}\right] \mathcal{B}^{2}, K=\mathcal{B}^{2} \max \left\{(3+\alpha)^{2}+2 \alpha^{2}, 2 \max \left\{9,3 \alpha^{2}\right\}\right\}, \\
& \hat{K}=\frac{\min \left\{32 x_{*}, x_{*}^{2} \alpha^{2} y_{*}\right\}}{16\left[K+\mathcal{B}^{2} \max \left\{9,3 \alpha^{2}\right\}\right]}, \bar{U}=\frac{\min \{\hat{K}, \theta\}}{4\left(\alpha \mathcal{B}^{3}+K \mathcal{B}\right)}, \\
& \text { and } \theta=\min \left\{\frac{K_{0} x_{*}^{2}}{8}, \frac{K_{0} x_{*}^{2} y_{*}^{2} \alpha^{2}}{8\left(x_{*}+2 \sqrt{K_{0}}\right)^{2}}\right\},
\end{aligned}
$$

where $\mathcal{B}$ is from (53). We continue to use the functions $V_{1}$ and $V_{2}$ from the preceding subsection. We prove the following (but see Remark 7 for results under integral ISS under a less stringent disturbance bound, and Section VII-B.3 for a specific numerical example):

Theorem 3: The system (54) is ISS with respect to disturbances $\mathbf{u}$ valued in $U=\bar{U} \mathcal{B}_{1}$.

Proof: The time derivatives of the functions $V_{1}$ and $V_{2}$ defined in Section VII-B, along the trajectories of 54 in $\mathcal{X}^{b}$, satisfy $\dot{V}_{1}=-\tilde{x}^{2}-\mathbf{u} \tilde{y} \leq-\tilde{x}^{2}+\mathcal{B}|\mathbf{u}|$ and

$$
\begin{aligned}
\dot{V}_{2} & =-(\tilde{x}+\alpha \tilde{y})^{2} x^{2}+\left\{-\tilde{x}\left(2 \tilde{x}+x_{*}\right)-\alpha \tilde{y} \tilde{x}\right\}(\tilde{x}+\alpha \tilde{y}) x+\tilde{x}\left[\alpha^{2} \tilde{x}-\alpha \mathbf{u}\right] x y \\
& \leq-\frac{1}{2}(\tilde{x}+\alpha \tilde{y})^{2} x^{2}+\frac{1}{2}\left\{\tilde{x}\left(2 \tilde{x}+x_{*}\right)+\alpha \tilde{y} \tilde{x}\right\}^{2}+\alpha^{2} \tilde{x}^{2} x y-\alpha \tilde{x} \mathbf{u} x y
\end{aligned}
$$

by the triangle inequality. Since $(x, y) \in \mathcal{S}$, we deduce that

$$
\begin{aligned}
\dot{V}_{2} & \leq-\frac{1}{2}(\tilde{x}+\alpha \tilde{y})^{2} x^{2}+\frac{(3+\alpha)^{2} \mathcal{B}^{2}}{2} \tilde{x}^{2}+\left[\alpha^{2} \tilde{x}^{2}+\alpha \mathcal{B}|\mathbf{u}|\right] \mathcal{B}^{2} \\
& \leq-\frac{1}{2}(\tilde{x}+\alpha \tilde{y})^{2} x^{2}+\left[\frac{(3+\alpha)^{2}}{2}+\alpha^{2}\right] \mathcal{B}^{2} \tilde{x}^{2}+\alpha \mathcal{B}^{3}|\mathbf{u}|
\end{aligned}
$$

On the other hand, according to $\mathrm{A} .6$ with the choices $A=\tilde{x} / x_{*}$ and then $A=\tilde{y} / y_{*}$, we have

$$
\tilde{x}-x_{*} \ln \left(1+\frac{\tilde{x}}{x_{*}}\right) \geq \frac{\tilde{x}^{2}}{2\left(2 x_{*}+x\right)} \text { and } \tilde{y}-y_{*} \ln \left(1+\frac{\tilde{y}}{y_{*}}\right) \geq \frac{\tilde{y}^{2}}{2\left(2 y_{*}+y\right)}
$$

and therefore

$$
V_{1}(\tilde{x}, \tilde{y}) \geq \frac{\tilde{x}^{2}}{2\left(2 x_{*}+x\right)}+\frac{\tilde{y}^{2}}{2\left(2 y_{*}+y\right)} \geq \frac{\tilde{x}^{2}+\tilde{y}^{2}}{6 \mathcal{B}}
$$


for all $(x, y) \in \mathcal{S}$. Moreover, for all $(x, y) \in \mathcal{S}$, we have

$$
\begin{aligned}
\left|V_{2}(\tilde{x}, \tilde{y})\right| & =|\tilde{x}[\tilde{x}+\alpha \tilde{y}] x| \leq\left(\tilde{x}^{2}+\alpha|\tilde{x} \tilde{y}|\right) \mathcal{B} \leq \mathcal{B}\left(\frac{3}{2} \tilde{x}^{2}+\frac{\alpha^{2}}{2} \tilde{y}^{2}\right) \\
& \leq \mathcal{B} \max \left\{\frac{3, \alpha^{2}}{2}\right\}\left(\tilde{x}^{2}+\tilde{y}^{2}\right) \leq \mathcal{B}^{2} \max \left\{9,3 \alpha^{2}\right\} V_{1}(\tilde{x}, \tilde{y})
\end{aligned}
$$

Let $\mathcal{U}_{K}(\tilde{x}, \tilde{y})=V_{2}(\tilde{x}, \tilde{y})+K V_{1}(\tilde{x}, \tilde{y})$. Then $\mathcal{U}_{K}(\tilde{x}, \tilde{y}) \geq\left[-\mathcal{B}^{2} \max \left\{9,3 \alpha^{2}\right\}+K\right] V_{1}(\tilde{x}, \tilde{y}) \geq$ $\mathcal{B}^{2} \max \left\{9,3 \alpha^{2}\right\} V_{1}(\tilde{x}, \tilde{y})$, by our choice of $K \geq K_{0}$ in $(56)$, and $\dot{\mathcal{U}}_{K} \leq-\mathcal{Q}(\tilde{x}, \tilde{y})+\overline{\mathcal{B}}|\mathbf{u}|$, where $\overline{\mathcal{B}}=\alpha \mathcal{B}^{3}+K \mathcal{B}$ and $\mathcal{Q}(\tilde{x}, \tilde{y})=\frac{1}{2}(\tilde{x}+\alpha \tilde{y})^{2} x^{2}+\frac{K_{0}}{2} \tilde{x}^{2}$. We consider two cases:

First case: $\mathcal{Q}(\tilde{x}, \tilde{y}) \geq \theta$. Then

$$
\dot{\mathcal{U}}_{K} \leq-\theta \frac{\mathcal{U}_{K}(\tilde{x}, \tilde{y})}{1+\mathcal{U}_{K}(\tilde{x}, \tilde{y})}+\overline{\mathcal{B}}|\mathbf{u}|
$$

Second case: $\mathcal{Q}(\tilde{x}, \tilde{y}) \leq \theta$. Then

$$
|\tilde{x}| \leq \sqrt{\frac{2}{K_{0}} \theta}
$$

and therefore the inequality $\theta \leq \frac{K_{0} x_{*}^{2}}{8}$ implies that

$$
|\tilde{x}| \leq \frac{x_{*}}{2}, \text { hence } \frac{x_{*}}{2} \leq x
$$

Moreover, $|\tilde{x}+\alpha \tilde{y}| x \leq \sqrt{2 \theta}$. It follows from 64 that

$$
|\tilde{x}+\alpha \tilde{y}| \leq \frac{2}{x_{*}} \sqrt{2 \theta}, \text { hence }|\tilde{y}| \leq \frac{2}{x_{*} \alpha} \sqrt{2 \theta}+\frac{1}{\alpha}|\tilde{x}|
$$

We deduce from (63) that

$$
|\tilde{y}| \leq \frac{2}{x_{*} \alpha} \sqrt{2 \theta}+\frac{1}{\alpha} \sqrt{\frac{2}{K_{0}} \theta} \leq \frac{\sqrt{2}\left(x_{*}+2 \sqrt{K_{0}}\right)}{\sqrt{K_{0}} x_{*} \alpha} \sqrt{\theta} \leq \frac{y_{*}}{2}
$$

by our choice of $\theta$.

Next, one can easily prove that for all $A \in\left[-\frac{1}{2}, \frac{1}{2}\right]$, we have $A-\ln (1+A) \leq A^{2}$, and therefore, 
when $|\tilde{x}| \leq \frac{x_{*}}{2}$ and $|\tilde{y}| \leq \frac{y_{*}}{2}$, we have

$$
V_{1}(\tilde{x}, \tilde{y}) \leq \frac{\tilde{x}^{2}}{x_{*}}+\frac{\tilde{y}^{2}}{y_{*}}
$$

Since the definition of $\mathcal{U}_{K}$ and 61 imply that $\mathcal{U}_{K}(\tilde{x}, \tilde{y}) \leq\left(K+\mathcal{B}^{2} \max \left\{9,3 \alpha^{2}\right\}\right) V_{1}(\tilde{x}, \tilde{y})$, we get

$$
\mathcal{U}_{K}(\tilde{x}, \tilde{y}) \leq \bar{K}\left[\frac{\tilde{x}^{2}}{x_{*}}+\frac{\tilde{y}^{2}}{y_{*}}\right]
$$

where $\bar{K}=K+\mathcal{B}^{2} \max \left\{9,3 \alpha^{2}\right\}$. Also, the inequality $x \geq \frac{x_{*}}{2}$ from 64 implies that

$$
\mathcal{Q}(\tilde{x}, \tilde{y}) \geq \frac{x_{*}^{2}}{8}(\tilde{x}+\alpha \tilde{y})^{2}+\frac{K_{0}}{2} \tilde{x}^{2}
$$

By separately considering the possibilities $|\tilde{x}| \geq \frac{1}{4} \alpha|\tilde{y}|$ and $|\tilde{x}| \leq \frac{1}{4} \alpha|\tilde{y}|$ and noting that $K_{0} \geq$ $9 \mathcal{B}^{2} \geq 9$, it follows from 69 that

$$
\mathcal{Q}(\tilde{x}, \tilde{y}) \geq \frac{x_{*}^{2}}{16} \alpha^{2} \tilde{y}^{2}+2 \tilde{x}^{2} \geq \min \left\{\frac{x_{*}^{2}}{16} \alpha^{2} y_{*}, 2 x_{*}\right\}\left[\frac{\tilde{x}^{2}}{x_{*}}+\frac{\tilde{y}^{2}}{y_{*}}\right]
$$

Combining (68) and (70) yields

$$
\mathcal{U}_{K}(\tilde{x}, \tilde{y}) \leq \bar{K} \frac{\mathcal{Q}(\tilde{x}, \tilde{y})}{\min \left\{2 x_{*}, \frac{x_{*}^{2}}{16} \alpha^{2} y_{*}\right\}}
$$

Recalling the estimate $\dot{\mathcal{U}}_{K} \leq-\mathcal{Q}(\tilde{x}, \tilde{y})+\overline{\mathcal{B}}|\mathbf{u}|$, we deduce that $\dot{\mathcal{U}}_{K} \leq-\widehat{K} \mathcal{U}_{K}(\tilde{x}, \tilde{y})+\overline{\mathcal{B}}|\mathbf{u}|$.

We deduce that, in both cases,

$$
\dot{\mathcal{U}}_{K} \leq-\mho \frac{\mathcal{U}_{K}(\tilde{x}, \tilde{y})}{1+\mathcal{U}_{K}(\tilde{x}, \tilde{y})}+\overline{\mathcal{B}}|\mathbf{u}|
$$

where $\mho=\min \{\widehat{K}, \theta\}$. Let $\overline{\mathcal{U}_{K}}(\tilde{x}, \tilde{y})=\mathcal{U}_{K}(\tilde{x}, \tilde{y}) e^{\mathcal{U}_{K}(\tilde{x}, \tilde{y})}$. Then

$$
\dot{\mathcal{U}_{K}}=e^{\mathcal{U}_{K}(\tilde{x}, \tilde{y})}\left[1+\mathcal{U}_{K}(\tilde{x}, \tilde{y})\right] \dot{\mathcal{U}}_{K} \leq e^{\mathcal{U}_{K}(\tilde{x}, \tilde{y})}\left[\{-\mho+\overline{\mathcal{B}}|\mathbf{u}|\} \mathcal{U}_{K}(\tilde{x}, \tilde{y})+\overline{\mathcal{B}}|\mathbf{u}|\right]
$$


Therefore, when $|\mathbf{u}|_{\infty} \leq \frac{\mho}{2 \overline{\mathcal{B}}}$, we have

$$
\begin{aligned}
\dot{\mathcal{U}_{K}} & \leq e^{\mathcal{U}_{K}(\tilde{x}, \tilde{y})}\left[-\frac{\mho}{2} \mathcal{U}_{K}(\tilde{x}, \tilde{y})+\overline{\mathcal{B}}|\mathbf{u}|\right] \leq-\frac{\mho}{2} \overline{\mathcal{U}_{K}}(\tilde{x}, \tilde{y})+\overline{\mathcal{B}}|\mathbf{u}| e^{\mathcal{U}_{K}(\tilde{x}, \tilde{y})} \\
& \leq-\frac{\mho}{2} \overline{\mathcal{U}_{K}}(\tilde{x}, \tilde{y})+\overline{\mathcal{B}}|\mathbf{u}|\left[e^{\mathcal{U}_{K}(\tilde{x}, \tilde{y})}-1\right]+\overline{\mathcal{B}}|\mathbf{u}| \\
& \leq-\frac{\mho}{2} \overline{\mathcal{U}_{K}}+\overline{\mathcal{B}}|\mathbf{u}| \overline{\mathcal{U}_{K}}+\overline{\mathcal{B}}|\mathbf{u}|
\end{aligned}
$$

where the last inequality used the condition $e^{a}-1 \leq a e^{a}$ for all $a \geq 0$. Therefore, when $\overline{\mathcal{B}}|\mathbf{u}| \leq \frac{\mho}{4}$, we obtain

$$
\dot{\overline{\mathcal{U}_{K}}} \leq-\frac{v}{4} \overline{\mathcal{U}_{K}}(\tilde{x}, \tilde{y})+\overline{\mathcal{B}}|\mathbf{u}|
$$

The desired ISS inequality now follows from standard arguments.

Remark 7: The Lyapunov function construction in the preceding proof can be used to explicitly construct the functions $\beta, \gamma$, and $\bar{\alpha}$ in the ISS estimate (55). The inequality (72) implies that $\mathcal{U}_{K}$ is an iISS Lyapunov function for the Lotka-Volterra errors dynamics (54) when the disturbance $\mathbf{u}$ satisfies the less stringent bound $|\mathbf{u}|_{\infty} \leq \frac{d}{2}$; see [19] for the original treatment of iISS, and see [2], [20] for extensive iISS discussions and results. In fact, a slight variant of the iISS arguments from [3] in conjunction with (72) and the growth properties of $\mathcal{U}_{K}$ can be used to explicitly construct functions $\beta \in \mathcal{K} \mathcal{L}$ and $\gamma \in \mathcal{K}_{\infty}$, a constant $\bar{G}>0$, and a modulus $\bar{\alpha}$ with respect to $\left(-x_{*}, \infty\right) \times\left(-y_{*}, \infty\right)$, such that for each disturbance $\mathbf{u}:[0, \infty) \rightarrow[-d / 2, d / 2]$ and each trajectory $(\tilde{x}, \tilde{y}):[0, \infty) \rightarrow \mathcal{X}^{b}$ of (54) corresponding to $\mathbf{u}$, we have

$$
\gamma(|(\tilde{x}, \tilde{y})(t)|) \leq \beta(\bar{\alpha}((\tilde{x}, \tilde{y})(0)), t)+\bar{G} \int_{0}^{t}|\mathbf{u}(r)| \mathrm{d} r \quad \forall t \geq 0
$$

We illustrate these ideas in the simulations below.

3) Simulations: To illustrate our findings, we simulated the dynamics (54) using the parameter values $\alpha=2, d=1, x_{*}=0.5$, and $y_{*}=0.25$, corresponding to the parameter choices

$$
a=\gamma=\beta=\Delta=0.5 \text { and } L=2
$$


in the original model. Hence, (54) is iISS with respect to disturbances that are bounded by 0.5 . We chose the disturbance $\mathbf{u}(t)=0.49 e^{-t}$. In Figures 1-2 below, we plotted the corresponding levels of $\zeta$ and $\chi$, which are related to $x$ and $y$ in terms of the coordinate changes (37).

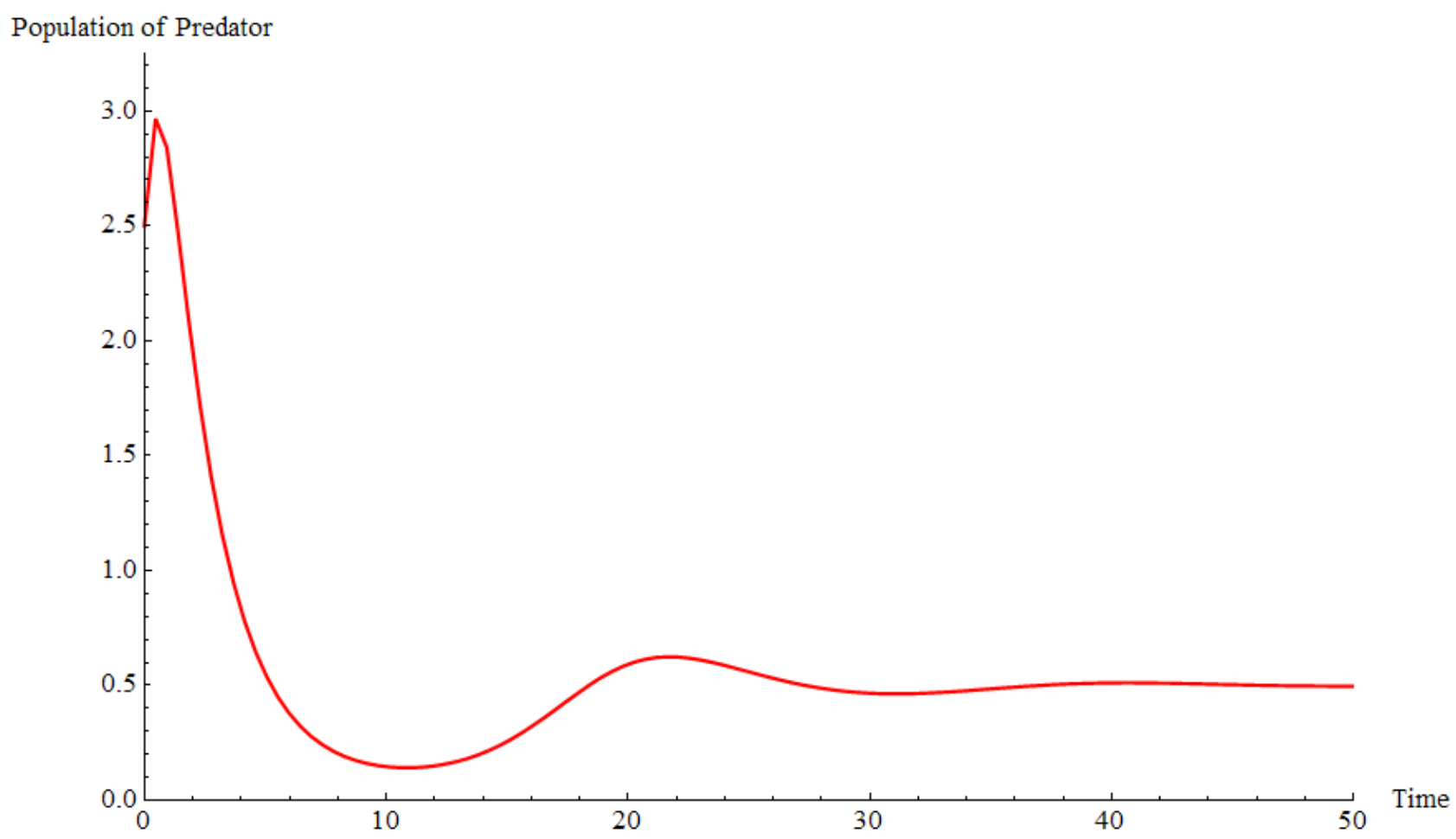

Fig. 1. Population of Predator $\zeta$ in Lotka-Volterra Dynamics 54 with Parameters 77 and $\mathbf{u}(t)=0.49 e^{-t}$

If $x(t) \rightarrow x_{*}=0.5$ and $y(t) \rightarrow y_{*}=0.25$, then the coordinate changes 37 give

$$
\zeta(t) \rightarrow 0.25 \frac{\beta L}{a}=0.5 \text { and } \chi(t) \rightarrow 0.5 L=1
$$

which is in fact the behavior we see in the figures. This shows the robustness of the convergence in the face of the disturbance $\mathbf{u}$.

\section{CONCLUSION}

We gave new methods for building global strict Lyapunov functions under LaSalle conditions. The novelty of our first result is in the generality of its assumptions. The novelty of our second 


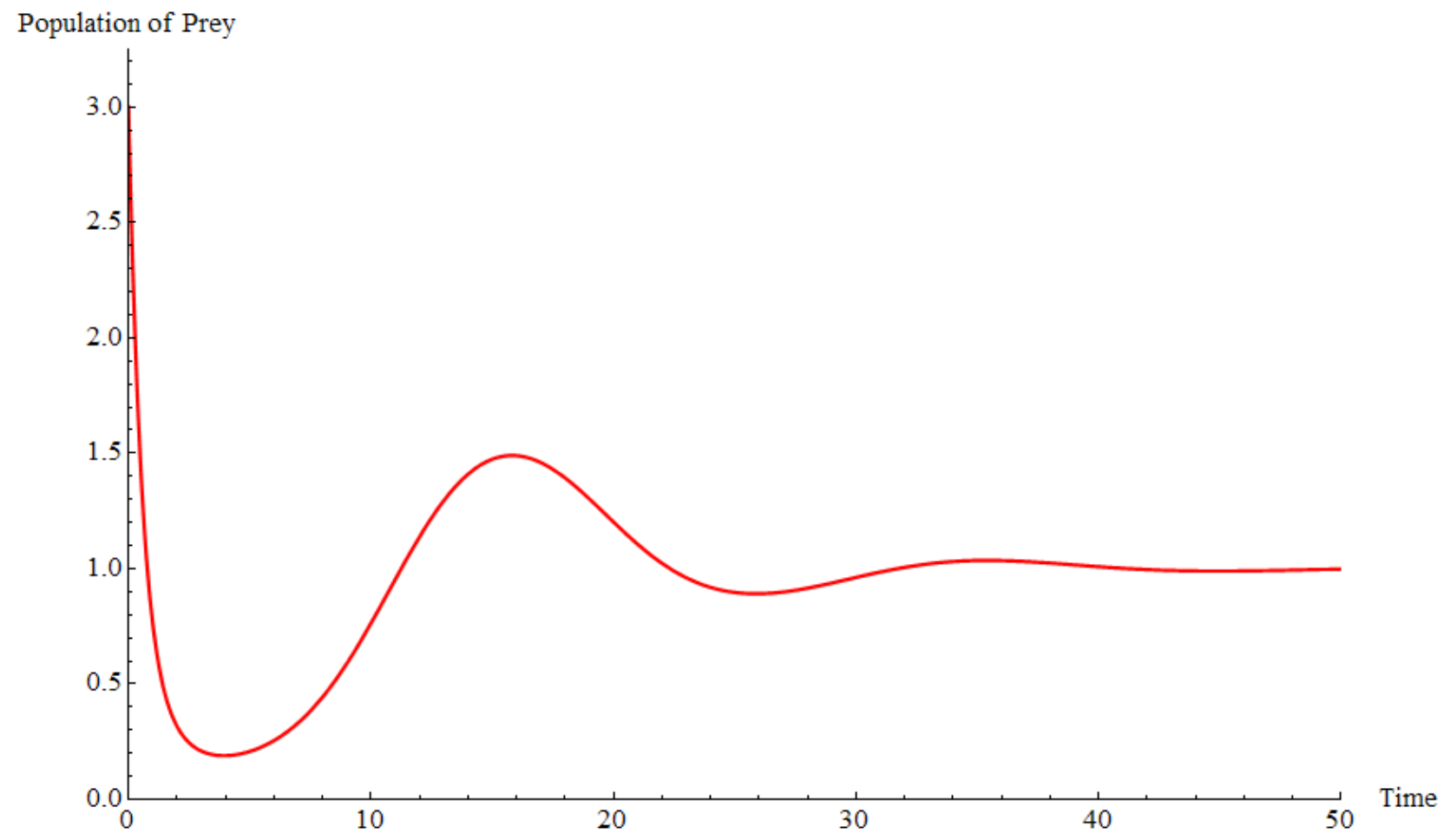

Fig. 2. Population of Prey $\chi$ in Lotka-Volterra Dynamics 54 with Parameters 77 and $\mathbf{u}(t)=0.49 e^{-t}$

one is in the local properties of our strict Lyapunov functions and its applicability on general state spaces. As a byproduct, we exhibited a general class of auxiliary functions for which the Matrosov theorem from [13] applies. We illustrated our work using a robustness analysis for the celebrated Lotka-Volterra model.

\section{A.1. A USEFul Lower Bound}

We used the following simple lemma on $\mathrm{p} .7$.

Lemma A.1: For each continuous positive definite function $\rho:[0, \infty) \rightarrow[0, \infty)$, we can find a function $\omega \in \mathcal{K}_{\infty} \cap C^{1}$ and a strictly increasing everywhere positive function $K \in C^{1}$ such that

$$
\rho(r) \geq \frac{\omega(r)}{K(r)}
$$

for all $r \geq 0$. 
Proof: By replacing $\rho$ with

$$
\rho_{\text {new }}(r)= \begin{cases}r \min \{\rho(q): r \leq q \leq 1\}, & 0 \leq r \leq 1 \\ \min \{\rho(q): 1 \leq q \leq r\}, & r>1\end{cases}
$$

without relabeling and noting that $\rho_{\text {new }}(r) \leq \rho(r)$ for all $r \geq 0$, we can assume that $\rho$ is strictly increasing on $[0,1]$ and nonincreasing on $[1, \infty)$. Notice that

$$
\rho(r)=\frac{\omega_{0}(r)}{K_{0}(r)}
$$

for all $r \geq 0$, where $\omega_{0}$ and $K_{0}$ are the increasing continuous functions

$$
\omega_{0}(r)=\left\{\begin{array}{ll}
\frac{\rho(r)}{\rho(1)}, & 0 \leq r \leq 1 \\
r, & r \geq 1
\end{array} \quad \text { and } K_{0}(r)= \begin{cases}\frac{1}{\rho(1)}, & 0 \leq r \leq 1 \\
\frac{r}{\rho(r)}, & r \geq 1\end{cases}\right.
$$

We can then satisfy A.1 by picking any function $\omega \in \mathcal{K}_{\infty} \cap C^{1}$ such that $\omega(r) \leq \omega_{0}(r)$ for all $r \geq 0$ and any strictly increasing $C^{1}$ function $K$ such that $K(r) \geq K_{0}(r)$ for all $r \geq 0$.

\section{A.2. Verifying Estimates 27)}

We only show how to satisfy the second estimate in (27); the other estimate in (27) is handled similarly. We maintain the notation from Section VI. Since $f(0)=0$, all of the functions $L_{f}^{i} h_{l}(x)$ are zero at the origin and sufficiently smooth for all $i \in \mathbb{N}$. Also, Assumption 1 provides a positive definite quadratic lower bound for $V_{1}$ near the origin. Moreover, the fact that $V_{1}$ is a storage function implies that there exists a function $\underline{\alpha} \in \mathcal{K}_{\infty}$ such that $V_{1}(x) \geq \underline{\alpha}(|x|)$ for all $x \in \mathcal{X}$. Hence,

$$
\sum_{l=1}^{m}\left|L_{f}^{i} h_{l}(x)\right| \leq|x| \mathcal{G}_{1}(|x|) \leq \bar{\kappa} \sqrt{V_{1}(x)} \mathcal{G}_{1}\left(\underline{\alpha}^{-1}\left(V_{1}(x)\right)\right) \quad \forall i \in\{3, \ldots, N\}
$$

for some increasing everywhere positive function $\mathcal{G}_{1}$ and some constant $\bar{\kappa}>0$ in some neighborhood $\mathcal{O}$ of the origin (by our choice of $\underline{\alpha}$ and the fact that $V_{1}$ is bounded from below by a positive definite 
quadratic function near 0 ). We can also find a $\mathcal{G}_{2} \in \mathcal{K}_{\infty}$ so that

$$
\frac{\sum_{l=1}^{m}\left|L_{f}^{i} h_{l}(x)\right|}{\sqrt{\underline{\alpha}(|x|)}} \leq \mathcal{G}_{2}(|x|) \quad \forall i \in\{3, \ldots, N\}
$$

on $\mathbb{R}^{n} \backslash \mathcal{O}$. Hence, we can take $\phi_{1}(r)=1+\bar{\kappa} \mathcal{G}_{1}\left(\underline{\alpha}^{-1}(r)\right)+\mathcal{G}_{2}\left(\underline{\alpha}^{-1}(r)\right)$.

\section{A.3. StRict LyapunOv FunCtion FOR (32)}

We construct the functions needed for the strict Lyapunov function construction for (32). We use the notation from Section VII-A and the proof of Theorem 1 . Then

$$
\begin{aligned}
\dot{a}_{3}= & -2 \sin (2 t) x_{1}^{2}+2(-3 \sin (t)+\cos (t)) x_{1} x_{2} \\
& +2(\sin (2 t)+\cos (t)[3 \cos (t)+\sin (t)]) x_{2}^{2} \\
& -2\left[(3 \cos (t)+\sin (t)) x_{1}+2\left(1+\sin ^{2}(t)\right) x_{2}\right]\left[\cos (t) x_{1}+x_{2}\right] .
\end{aligned}
$$

Applying the relation $\left|x_{1} x_{2}\right| \leq \frac{1}{2} x_{1}^{2}+x_{2}^{2}$ and collecting coefficients of $x_{1}^{2}$ and $x_{2}^{2}$ readily gives $\max \left\{\left|a_{i}(t, x)\right|: t \in \mathbb{R}, 1 \leq i \leq 4\right\} \leq 60 V(x)$, so $\Gamma(r)=300 r+1$ satisfies our requirements. Since $\ell=3, T=2 \pi$, and $\tau=\frac{\pi}{4}$, taking $\omega(r)=r^{2}$ and $K(r)=200(r+1)$ gives

$$
\begin{aligned}
& \Omega(v)=\frac{2 \tau \omega(v)}{3 T(\ell-2) \Gamma^{2}(v) K(v)}=\frac{v^{2}}{2400(v+1)(300 v+1)^{2}}, \text { hence } \\
& k_{2}(v)=\omega^{2^{\ell-1}}(v)=v^{8} \text { and } k_{1}(v)=\frac{k_{2}(v)}{\Omega(v)}=2400 v^{6}(v+1)(300 v+1)^{2} .
\end{aligned}
$$

Since $\omega(v)=v^{2}$, our requirement 14 on $G$ is satisfied if $G^{\prime}(v) \geq \frac{2 \pi}{K(v)}\left(k_{2}(v)\left[2 v K(v)-200 v^{2}\right]+\right.$ $\left.k_{2}^{\prime}(v) v^{2}\right)$ for all $v \geq 0$, which holds if $G(v)=5 \pi v^{9}$.

Since $\left|M_{1}(t, x)\right|=\left|a_{1}(t, x) a_{2}(t, x)+150 V^{2}(x)+V(x)\right| \leq 162 V^{2}(x)+V(x)$ and $\left|M_{2}(t, x)\right|=$ $\left|a_{1}(t, x) a_{2}(t, x)+a_{2}(t, x) a_{3}(t, x)+150 V^{2}(x)+V(x)\right| \leq 306 V^{2}(x)+V(x)$, we can satisfy (13) using $k_{0}(v)=\left(k_{1}^{\prime}(v)+k_{2}^{\prime}(v)\right)\left(306 v^{2}+v\right)+1$. Also, $\left|S_{2}(t, x)\right| \leq G(V(x))+2 \pi V^{10}(x)$ and $\left|S_{1}(t, x)\right| \leq$ $\max \left\{k_{1}(V(x)), k_{2}(V(x))\right\}\left[306 V^{2}(x)+V(x)\right]+k_{0}(V(x)) V(x)$. Therefore, we can take $\kappa(v)=$ $\left[k_{1}(v)+k_{2}(v)\right]\left(306 v^{2}+v\right)+k_{0}(v) v+G(v)+2 \pi v^{10}+1$. The formula for $V^{\sharp}$ is now immediate from plugging in the preceding functions into the construction $(10)$ from Theorem 1 . 


\section{A.4. UsEFul INEQUALITIES}

We used the following simple lemma in Section VII-B;

Lemma A.2: For all $A \in(-1, \infty)$, the following inequalities hold:

$$
A-\ln (1+A) \geq \frac{A^{2}}{2(1+|A|)} \quad \text { and } \quad|A| \leq 2 \sqrt{[A-\ln (1+A)]+[A-\ln (1+A)]^{2}}
$$

Proof: First assume that $A \in(-1,0)$. Then

$$
A-\ln (1+A)=\int_{0}^{A} \frac{m}{1+m} \mathrm{~d} m \geq \frac{A^{2}}{2} .
$$

If on the other hand $A \geq 0$, then

$$
A-\ln (1+A)=\int_{0}^{A} \frac{m}{1+m} \mathrm{~d} m \geq \int_{0}^{A} \frac{m}{1+A} \mathrm{~d} m=\frac{A^{2}}{2(1+A)}
$$

which gives the first condition A.6.

To prove the second condition in $($ A.6), notice that the first condition implies that for all $A>-1$,

$$
2[A-\ln (1+A)]+2|A|[A-\ln (1+A)] \geq A^{2}
$$

Applying the triangle inequality $c_{1} c_{2} \leq \frac{c_{1}^{2}}{2}+\frac{c_{2}^{2}}{2}$ gives $\{|A|\}\{2[A-\ln (1+A)]\} \leq \frac{1}{2} A^{2}+2[A-\ln (1+$ $A)]^{2}$, which we can combine with $\mathrm{A.7}$ to deduce that $2 \sqrt{[A-\ln (1+A)]+[A-\ln (1+A)]^{2}} \geq$ $|A|$. This gives A.6.

\section{A.5. VERIFYING (44) FOR LOTKA-VOLTERRA EXAMPLE}

We show that (44) is satisfied for an appropriate choice of the constant $\underline{d}>0$. We continue to use the notation of Section VII-B. Consider the function

$$
E(p, q)=p-q \ln \left(1+\frac{p}{q}\right)
$$


which is defined for all $p>-q$ when $q>0$. For each $q>0$,

$$
E(p, q) \rightarrow \infty \text { as } p \rightarrow-q^{+}
$$

Also, our choice 42 of $V_{1}$ says $V_{1}(\tilde{x}, \tilde{y})=E\left(\tilde{x}, x_{*}\right)+E\left(\tilde{y}, y_{*}\right)$. Setting $\mathcal{N}_{1}(\tilde{x}, \tilde{y})=\frac{1}{2} h_{1}(\tilde{x}, \tilde{y})^{2}$ and $\mathcal{N}_{2}(\tilde{x}, \tilde{y})=\left[L_{f} h_{1}(\tilde{x}, \tilde{y})\right]^{2}$ as before, we claim that we can find a constant

$$
\delta \in\left(0, \frac{1}{2} \min \left\{x_{*}, y_{*}\right\}\right]
$$

so that

$$
\sum_{i=1}^{2} \mathcal{N}_{i}(\tilde{x}, \tilde{y})=\frac{1}{2} \tilde{x}^{2}+\left[(\tilde{x}+\alpha \tilde{y})\left(\tilde{x}+x_{*}\right)\right]^{2} \geq \frac{\delta^{2} V_{1}(\tilde{x}, \tilde{y})}{1+V_{1}^{2}(\tilde{x}, \tilde{y})}
$$

for all $(\tilde{x}, \tilde{y})$ in the set $\mathcal{D}=\left\{(\tilde{x}, \tilde{y}) \in \mathcal{X}: \tilde{x} \leq-x_{*}+\delta\right.$ or $\left.\tilde{y} \leq-y_{*}+\delta\right\}$. To check this claim, first note that for any $\delta$ satisfying $\left(\right.$ A.9,$\sum_{i=1}^{2} \mathcal{N}_{i}(\tilde{x}, \tilde{y})$ is bounded from below on $\mathcal{D}$ by a positive constant depending on $\delta$. (Indeed, if $\tilde{x} \leq-x_{*}+\delta$, then $\sum_{i=1}^{2} \mathcal{N}_{i}(\tilde{x}, \tilde{y}) \geq \frac{1}{8} x_{*}^{2}$. If on the other hand $\tilde{x} \geq-x_{*}+\delta$, then $\sum_{i=1}^{2} \mathcal{N}_{i}(\tilde{x}, \tilde{y}) \geq \frac{1}{2} \delta^{2} \tilde{x}^{2}+\delta^{2}(\tilde{x}+\alpha \tilde{y})^{2}$, since $\delta \leq x_{*}<1$. Since $\alpha>0$, $\frac{1}{2} \tilde{x}^{2}+(\tilde{x}+\alpha \tilde{y})^{2}$ is a positive definite quadratic form and so admits a constant $c_{*} \in(0,1)$ so that $\frac{1}{2} \tilde{x}^{2}+(\tilde{x}+\alpha \tilde{y})^{2} \geq c_{*}\left(\tilde{x}^{2}+\tilde{y}^{2}\right)$ on $\mathbb{R}^{2}$, which is bounded below by $c_{*} y_{*}^{2} / 4$ when $\tilde{y} \leq-y_{*}+\delta$. Hence,

$$
\sum_{i=1}^{2} \mathcal{N}_{i}(\tilde{x}, \tilde{y}) \geq \delta^{2} \frac{c_{*}}{8} \min \left\{x_{*}^{2}, y_{*}^{2}\right\}=: \underline{m}(\delta)
$$

on $\mathcal{D}$.) Reducing $\delta>0$ and recalling (A.8) guarantees that

$$
\frac{\delta^{2} V_{1}(\tilde{x}, \tilde{y})}{1+V_{1}^{2}(\tilde{x}, \tilde{y})} \leq \underline{m}(\delta)
$$

on $\mathcal{D}$. The claim now follows by combining (A.11)-A.12). Fix a constant $\delta>0$ satisfying the preceding requirements.

We next consider points in $\mathcal{X} \backslash \mathcal{D}$. For each constant $q>0$, we can find a constant $c(q)>1$ 
such that

$$
E(p, q) \leq c(q) p^{2} \quad \forall p \geq-q+\delta
$$

by applying L'Hôpital's rule to $E(p, q) / p^{2}$ as $p \rightarrow 0$ or $p \rightarrow \infty$. Therefore,

$$
V_{1}(\tilde{x}, \tilde{y})=\tilde{x}^{2}\left(\frac{E\left(\tilde{x}, x_{*}\right)}{\tilde{x}^{2}}\right)+\tilde{y}^{2}\left(\frac{E\left(\tilde{y}, y_{*}\right)}{\tilde{y}^{2}}\right) \leq\left[c\left(x_{*}\right)+c\left(y_{*}\right)\right]\left(\tilde{x}^{2}+\tilde{y}^{2}\right)
$$

on $\mathcal{X} \backslash \mathcal{D}$ when neither $\tilde{x}$ nor $\tilde{y}$ is zero. Similar reasoning gives

$$
V_{1}(\tilde{x}, \tilde{y}) \leq\left[c\left(x_{*}\right)+c\left(y_{*}\right)\right]\left(\tilde{x}^{2}+\tilde{y}^{2}\right)
$$

on all of $\mathcal{X} \backslash \mathcal{D}$ (by separately considering the possibilities $\tilde{x}=0$ and $\tilde{x} \neq 0$ and similarly for $\tilde{y})$. Moreover, we can find a constant $\underline{c}>0$ so that $\mathcal{N}_{1}(\tilde{x}, \tilde{y})+\mathcal{N}_{2}(\tilde{x}, \tilde{y}) \geq \underline{c}\left(\tilde{x}^{2}+\tilde{y}^{2}\right)$ on $\mathcal{X} \backslash \mathcal{D}$, because $\left(\tilde{x}+x_{*}\right)^{2} \geq \delta^{2}$ on $\mathcal{X} \backslash \mathcal{D}$ and $\frac{1}{2} \tilde{x}^{2}+\delta^{2}[\tilde{x}+\alpha \tilde{y}]^{2}$ is a positive definite quadratic function (again using the fact that $\alpha>0$ ). Therefore,

$$
\sum_{i=1}^{2} \mathcal{N}_{i}(\tilde{x}, \tilde{y}) \geq\left(\frac{\underline{c}}{c\left(x_{*}\right)+c\left(y_{*}\right)}\right)\left[c\left(x_{*}\right)+c\left(y_{*}\right)\right]\left(\tilde{x}^{2}+\tilde{y}^{2}\right) \geq\left(\frac{\underline{c}}{c\left(x_{*}\right)+c\left(y_{*}\right)}\right) V_{1}(\tilde{x}, \tilde{y})
$$

on $\mathcal{X} \backslash \mathcal{D}$. It follows from $\mathrm{A} .10]$ and $(\mathrm{A} .13)$ that we can take

$$
\underline{d}=\min \left\{\frac{\underline{c}}{c\left(x_{*}\right)+c\left(y_{*}\right)}, \delta^{2}\right\}
$$

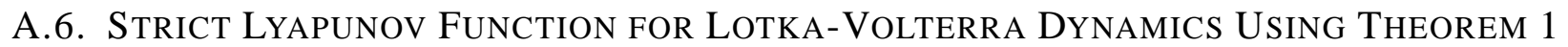

To further illustrate Theorem 1, we show how it applies to the Lotka-Volterra dynamics, after a change of variables. We take the change of coordinates $\xi=\ln (x)$ and $\psi=\ln (y)$. Taking $x_{*}=\frac{d}{\alpha} \in(0,1)$ and $y_{*}=\frac{1-x_{*}}{\alpha}>0$ as before, we also set $\xi_{*}=\ln \left(x_{*}\right)$ and $\psi_{*}=\ln \left(y_{*}\right)$. This and (38) give the dynamics

$$
\left\{\begin{aligned}
\dot{\tilde{\xi}} & =x_{*}\left[1-e^{\tilde{\xi}}\right]+\theta_{1}\left[1-e^{\tilde{\psi}}\right] \\
\dot{\tilde{\psi}} & =\theta_{2}\left[e^{\tilde{\xi}}-1\right]
\end{aligned}\right.
$$


for the error variables $\tilde{\xi}=\xi-\xi_{*}$ and $\tilde{\psi}=\psi-\psi_{*}$, where $\theta_{1}=\alpha y_{*}$ and $\theta_{2}=\alpha x_{*}$. The state space for $\mathrm{A} .15)$ is $\mathbb{R}^{2}$. We show how $\left.\mathrm{A} .15\right)$ is covered by Theorem 1 . Due to space constraints, we do not construct the strict Lyapunov function for $(\mathrm{A} .15)$ from Theorem 1 , since we already constructed the strict Lyapunov function (52) for the Lotka-Volterra error dynamics using Theorem 2

Let

$$
V(\tilde{\xi}, \tilde{\psi})=\theta_{2}\left[e^{\tilde{\xi}}-1-\tilde{\xi}\right]+\theta_{1}\left[e^{\tilde{\psi}}-1-\tilde{\psi}\right]
$$

Then $V$ is a storage function whose time derivative along the trajectories of (A.15) satisfies

$$
\dot{V}=\theta_{2}\left[e^{\tilde{\xi}}-1\right]\left[x_{*}\left(1-e^{\tilde{\xi}}\right)+\theta_{1}\left(1-e^{\tilde{\psi}}\right)\right]+\theta_{1}\left[e^{\tilde{\psi}}-1\right] \theta_{2}\left[e^{\tilde{\xi}}-1\right]=-\theta_{3}\left[e^{\tilde{\xi}}-1\right]^{2},
$$

where $\theta_{3}=\theta_{2} x_{*}$, so $V$ is a nonstrict Lyapunov function for the error dynamics A.15). Also, $a_{1}=\theta_{3}\left[e^{\tilde{\xi}}-1\right]^{2} \geq 0$. Taking $a_{2}=-\dot{a}_{1}$ as before gives

$$
\begin{aligned}
\dot{a}_{1}= & 2 \theta_{3} e^{\tilde{\xi}}\left[-x_{*}\left(1-e^{\tilde{\xi}}\right)^{2}-\theta_{1}\left(e^{\tilde{\xi}}-1\right)\left(e^{\tilde{\psi}}-1\right)\right] \text { and } \\
\dot{a}_{2}= & 2 \theta_{3} e^{\tilde{\xi} \dot{\tilde{\xi}}}\left[x_{*}\left(1-e^{\tilde{\xi}}\right)^{2}+\theta_{1}\left(e^{\tilde{\xi}}-1\right)\left(e^{\tilde{\psi}}-1\right)\right] \\
& +2 \theta_{3} e^{\tilde{\xi}}\left[2 x_{*}\left(e^{\tilde{\xi}}-1\right) e^{\tilde{\xi} \dot{\xi}}+\theta_{1}\left(e^{\tilde{\psi}}-1\right) e^{\tilde{\xi} \dot{\tilde{\xi}}}+\theta_{1}\left(e^{\tilde{\xi}}-1\right) e^{\tilde{\psi}} \dot{\tilde{\psi}}\right] .
\end{aligned}
$$

Therefore,

$$
\begin{aligned}
\frac{\dot{a}_{2}}{2 \theta_{3} e^{\tilde{\xi}}}= & \dot{\tilde{\xi}}\left[x_{*}\left(1-e^{\tilde{\xi}}\right)^{2}+\theta_{1}\left(e^{\tilde{\xi}}-1\right)\left(e^{\tilde{\psi}}-1\right)\right]+2 x_{*}\left(e^{\tilde{\xi}}-1\right) e^{\tilde{\xi} \dot{\tilde{\xi}}} \\
& +\theta_{1}\left(e^{\tilde{\psi}}-1\right) e^{\tilde{\xi} \dot{\tilde{\xi}}}+\theta_{1}\left(e^{\tilde{\xi}}-1\right) e^{\tilde{\psi} \dot{\tilde{\psi}}} \\
= & \dot{\tilde{\xi}}\left[\frac{a_{2}}{2 \theta_{3} e^{\tilde{\xi}}}+2 x_{*}\left(e^{\tilde{\xi}}-1\right) e^{\tilde{\xi}}\right]+\theta_{1} \theta_{2}\left(e^{\tilde{\xi}}-1\right)^{2} e^{\tilde{\psi}} \\
& +\theta_{1}\left(e^{\tilde{\psi}}-1\right) e^{\tilde{\xi}}\left[x_{*}\left(1-e^{\tilde{\xi}}\right)+\theta_{1}\left(1-e^{\tilde{\psi}}\right)\right] \\
= & {\left[x_{*}\left(1-e^{\tilde{\xi}}\right)+\theta_{1}\left(1-e^{\tilde{\psi}}\right)\right]\left[\frac{a_{2}}{2 \theta_{3} e^{\tilde{\xi}}}+2 x_{*}\left(e^{\tilde{\xi}}-1\right) e^{\tilde{\xi}}\right]+\theta_{1} \theta_{2}\left(e^{\tilde{\xi}}-1\right)^{2} e^{\tilde{\psi}} } \\
& +x_{*} \theta_{1} e^{\tilde{\xi}}\left(e^{\tilde{\psi}}-1\right)\left(1-e^{\tilde{\xi}}\right)-\theta_{1}^{2}\left(e^{\tilde{\psi}}-1\right)^{2} e^{\tilde{\xi}} .
\end{aligned}
$$


Then

$$
\begin{aligned}
\dot{a}_{2}= & {\left[x_{*}\left(1-e^{\tilde{\xi}}\right)+\theta_{1}\left(1-e^{\tilde{\psi}}\right)\right]\left[a_{2}+4 \theta_{3} x_{*}\left(e^{\tilde{\xi}}-1\right) e^{2 \tilde{\xi}}\right]+2 \theta_{1} \theta_{2} a_{1} e^{\tilde{\xi}} e^{\tilde{\psi}} } \\
& +2 \theta_{3} x_{*} \theta_{1} e^{2 \tilde{\xi}}\left(e^{\tilde{\psi}}-1\right)\left(1-e^{\tilde{\xi}}\right)-2 \theta_{1}^{2} \theta_{3}\left(e^{\tilde{\psi}}-1\right)^{2} e^{2 \tilde{\xi}} \\
= & {\left[x_{*}\left(1-e^{\tilde{\xi}}\right)+\theta_{1}\left(1-e^{\tilde{\psi}}\right)\right] a_{2}+\left[-4 x_{*}^{2} e^{2 \tilde{\xi}}+2 \theta_{1} \theta_{2} e^{\tilde{\xi}} e^{\tilde{\psi}}\right] a_{1} } \\
& +6 \theta_{3} x_{*} \theta_{1} e^{2 \tilde{\xi}}\left(e^{\tilde{\psi}}-1\right)\left(1-e^{\tilde{\xi}}\right)-2 \theta_{1}^{2} \theta_{3}\left(e^{\tilde{\psi}}-1\right)^{2} e^{2 \tilde{\xi}} .
\end{aligned}
$$

Taking $a_{3}=-\dot{a}_{2}$ as before, it readily follows that $\mathcal{M}(\tilde{\xi}, \tilde{\psi}):=a_{1}(\tilde{\xi}, \tilde{\psi})+a_{2}^{2}(\tilde{\xi}, \tilde{\psi})+a_{3}^{2}(\tilde{\xi}, \tilde{\psi})$ is positive definite, so (A.15) satisfies the assumptions of Theorem 1

\section{A.7. ACKNOWLEDGEMENT}

The first author acknowledges enlightening discussions with D. Nešić about the importance of local properties of Lyapunov functions.

\section{REFERENCES}

[1] D. Angeli. Input-to-state stability of PD-controlled robotic systems. Automatica J. IFAC, 35(7):1285-1290, 1999.

[2] D. Angeli, B. Ingalls, E.D. Sontag, and Y. Wang. Separation principles for input-output and integral-input to state stability. SIAM J. Control Optim., 43(1):256-276, 2004.

[3] D. Angeli, E.D. Sontag, and Y. Wang. A characterization of integral input-to-state stability. IEEE Trans. Automat. Contr, 45:1082-1097, June 2000.

[4] F. Clarke, Yu. Ledyaev, and R. Stern. Asymptotic stability and smooth Lyapunov functions. J. Differential Equations, 149(1):69$114,1998$.

[5] L. Faubourg and J-B. Pomet. Control Lyapunov functions for homogeneous "Jurdjevic-Quinn" systems. ESAIM Control Optim. Calc. Var., 5:293-311, 2000.

[6] S. Hsu. Limiting behavior for competing species. SIAM J. Appl. Math, 34(4):760-763, 1978.

[7] V. Jurdjevic and J.P. Quinn. Controllability and stability. J. Differential Equations, 28(3):381-389, 1978.

[8] H. Khalil. Nonlinear Systems, Third Edition. Prentice Hall, Upper Saddle River, NJ, 2002.

[9] M. Krstić, I. Kanellakopoulos, and P. Kokotovic. Nonlinear and Adaptive Control Design. Wiley, New York, 1995.

[10] A. Leung. Limiting behavior for several interacting populations. Math. Biosci., 29(1-2):85-98, 1976.

[11] A. Loría, E. Panteley, D. Popović, and A. Teel. A nested Matrosov theorem and persistency of excitation for uniform convergence in stable nonautonomous systems. IEEE Trans. Automat. Contr., 50:183-198, February 2005. 
[12] F. Mazenc and M. Malisoff. Further constructions of control-Lyapunov functions and stabilizing feedbacks for systems satisfying the Jurdjevic-Quinn conditions. IEEE Trans. Automat. Contr., 51:360-365, February 2006.

[13] F. Mazenc, M. Malisoff, and O. Bernard. A simplified design for strict Lyapunov functions under Matrosov conditions. IEEE Trans. Automat. Contr., 54:177-183, January 2009.

[14] F. Mazenc and D. Nešić. Strong Lyapunov functions for systems satisfying the conditions of La Salle. IEEE Trans. Automat. Contr., 49:1026-1030, June 2004.

[15] F. Mazenc and L. Praly. Adding integrations, saturated controls, and stabilization for feedforward systems. IEEE Trans. Automat. Contr., 41:1559-1578, November 1996.

[16] R. Outbib and J. Vivalda. On the stabilization of smooth nonlinear systems. IEEE Trans. Automat. Contr., 44:200-202, January 1999.

[17] R. Sepulchre, M. Janković, and P. V. Kokotović. Constructive Nonlinear Control. Springer-Verlag, Berlin, 1997.

[18] E.D. Sontag. A “universal” construction of Artstein's theorem on nonlinear stabilization. Systems Control Lett., 13(2):117-123, 1989.

[19] E.D. Sontag. Comments on integral variants of ISS. Systems and Control Letters, 34(1-2):93-100, 1998.

[20] E.D. Sontag. Input-to-state stability: Basic concepts and results. In P. Nistri and G. Stefani, editors, Nonlinear and Optimal Control Theory. Lectures Given at the C.I.M.E. Summer School Held in Cetraro, Italy June 19-29, 2004, volume 1932 of Lecture Notes in Mathematics, pages 163-220. Springer, Berlin, Germany, 2008. 